\title{
The ALMA-PILS survey: the sulphur connection between protostars and comets: IRAS 16293-2422 B and 67P/Churyumov-Gerasimenko
}

\author{
Maria N. Drozdovskaya, ${ }^{1 \star}$ Ewine F. van Dishoeck, ${ }^{2,3}$ Jes K. Jørgensen, ${ }^{4}$ \\ Ursina Calmonte, ${ }^{5}$ Matthijs H. D. van der Wiel, ${ }^{6}$ Audrey Coutens, ${ }^{7}$ Hannah Calcutt, ${ }^{4}$ \\ Holger S. P. Müller, ${ }^{8}$ Per Bjerkeli, ${ }^{9}$ Magnus V. Persson, ${ }^{9}$ Susanne F. Wampfler ${ }^{1}$ \\ and Kathrin Altwegg ${ }^{5}$ \\ ${ }^{1}$ Center for Space and Habitability, Universität Bern, Sidlerstrasse 5, CH-3012 Bern, Switzerland \\ ${ }^{2}$ Leiden Observatory, Leiden University, PO Box 9513, NL-2300 RA Leiden, the Netherlands \\ ${ }^{3}$ Max-Planck-Institut für Extraterrestrische Physik, Giessenbachstrasse 1, D-85748 Garching, Germany \\ ${ }^{4}$ Centre for Star and Planet Formation, Niels Bohr Institute \& Natural History Museum of Denmark, University of Copenhagen, Øster Voldgade 5-7, \\ DK-1350 Copenhagen K., Denmark \\ ${ }^{5}$ Physikalisches Institut, Universität Bern, Sidlerstrasse 5, CH-3012 Bern, Switzerland \\ ${ }^{6}$ ASTRON, The Netherlands Institute for Radio Astronomy, Postbus 2, NL-7990 AA Dwingeloo, the Netherlands \\ ${ }^{7}$ Laboratoire d'Astrophysique de Bordeaux, Univ. Bordeaux, CNRS, B18N, allée Geoffroy Saint-Hilaire, F-33615 Pessac, France \\ ${ }^{8}$ I. Physikalisches Institut, Universität zu Köln, Zülpicher Strasse 77, D-50937 Köln, Germany \\ ${ }^{9}$ Department of Space, Earth and Environment, Chalmers University of Technology, Onsala Space Observatory, SE-439 92 Onsala, Sweden
}

Accepted 2018 February 1. Received 2018 January 26; in original form 2017 September 8

\begin{abstract}
The evolutionary past of our Solar system can be pieced together by comparing analogous lowmass protostars with remnants of our Protosolar Nebula - comets. Sulphur-bearing molecules may be unique tracers of the joint evolution of the volatile and refractory components. ALMA Band 7 data from the large unbiased Protostellar Interferometric Line Survey are used to search for S-bearing molecules in the outer disc-like structure, $\sim 60$ au from IRAS 16293-2422 B, and are compared with data on 67P/Churyumov-Gerasimenko $(67 \mathrm{P} / \mathrm{C}-\mathrm{G})$ stemming from the ROSINA (Rosetta Orbiter Spectrometer for Ion and Neutral Analysis) instrument aboard Rosetta. Species such as $\mathrm{SO}_{2}, \mathrm{SO}, \mathrm{OCS}, \mathrm{CS}, \mathrm{H}_{2} \mathrm{CS}, \mathrm{H}_{2} \mathrm{~S}$, and $\mathrm{CH}_{3} \mathrm{SH}$ are detected via at least one of their isotopologues towards IRAS 16293-2422 B. The search reveals a first-time detection of $\mathrm{OC}^{33} \mathrm{~S}$ towards this source and a tentative first-time detection of $\mathrm{C}^{36} \mathrm{~S}$ towards a low-mass protostar. The data show that IRAS 16293-2422 B contains much more OCS than $\mathrm{H}_{2} \mathrm{~S}$ in comparison to $67 \mathrm{P} / \mathrm{C}-\mathrm{G}$; meanwhile, the $\mathrm{SO} / \mathrm{SO}_{2}$ ratio is in close agreement between the two targets. IRAS $16293-2422 \mathrm{~B}$ has a $\mathrm{CH}_{3} \mathrm{SH} / \mathrm{H}_{2} \mathrm{CS}$ ratio in range of that of our Solar system (differences by a factor of 0.7-5.3). It is suggested that the levels of UV radiation during the initial collapse of the systems may have varied and have potentially been higher for IRAS 16293-2422 B due to its binary nature; thereby, converting more $\mathrm{H}_{2} \mathrm{~S}$ into OCS. It remains to be conclusively tested if this also promotes the formation of S-bearing complex organics. Elevated UV levels of IRAS 16293-2422 B and a warmer birth cloud of our Solar system may jointly explain the variations between the two low-mass systems.
\end{abstract}

Key words: astrochemistry-comets: general-stars: protostars-ISM: molecules.

\section{INTRODUCTION}

Sulphur-bearing molecules have been detected in many interstellar environments from diffuse clouds to star-forming regions and rocky

^E-mail: maria.drozdovskaya@csh.unibe.ch bodies in our Solar system. It is likely that the sulphur detected in cometary studies has its roots in the earliest diffuse phases of the interstellar medium (ISM; e.g. Lucas \& Liszt 2002). However, in comparison to diffuse clouds, observations show the total sulphur budget of dense cores to be depleted by several orders of magnitude (Ruffle et al. 1999). This puzzle remains unresolved, and the missing sulphur is yet to be conclusively identified (e.g. Anderson et al. 
2013). Such depletion is unique to sulphur, making it a key element in understanding the evolution of volatile and refractory components between the diffuse and dense phases.

Some of the first detections of interstellar sulphur came in the 1970s and 1980s when molecules such as CS, OCS, $\mathrm{H}_{2} \mathrm{~S}$, SO, $\mathrm{H}_{2} \mathrm{CS}$, and $\mathrm{SO}_{2}$ were observed in the gas phase towards Sgr B2 and Orion A (Jefferts et al. 1971; Penzias et al. 1971; Drdla, Knapp \& van Dishoeck 1989; Minh et al. 1990; Pastor et al. 1991). These detections kicked off the study of its chemistry, which suggested that in thin clouds, sulphur is predominantly in the form of $\mathrm{S}^{+}$ ions (Oppenheimer \& Dalgarno 1974) and likely undergoes partial incorporation into refractories. Meanwhile, in dense clouds it is mostly neutral and gets incorporated into the observed volatiles via reactions with $\mathrm{H}_{3}^{+}$(Oppenheimer \& Dalgarno 1974) and grainsurface reactions (Duley, Millar \& Williams 1980). Subsequent chemical models quickly showed that the net sulphur budget as seen in volatiles is severely depleted in dense clouds (Prasad \& Huntress 1982). Some of the sulphur is incorporated into carbon chains such as $\mathrm{C}_{n} \mathrm{~S}$ (with $n=1,2, \ldots$; Smith et al. 1988; Wlodek, Bohme \& Herbst 1988; Millar \& Herbst 1990; Hirahara et al. 1992). However, since the ionization potential of sulphur is lower than that of carbon, $\mathrm{S}^{+}$may exist in regions where most of carbon is neutral, which may lead to the formation of $S_{2}$ (suggested early on by Duley et al. 1980). In photodissociation regions, it appears that sulphur is found in the form of CS and $\mathrm{HCS}^{+}$(Jansen, van Dishoeck \& Black 1994; Goicoechea et al. 2006).

Sulphur-bearing species are also seen in solid form: OCS ice was the first to be detected (Palumbo, Tielens \& Tokunaga 1995; Palumbo, Geballe \& Tielens 1997; Aikawa et al. 2012) and the detection of solid $\mathrm{SO}_{2}$ followed (Boogert et al. 1997). These ices have been seen in absorption against the bright high-mass W33A protostar and account for $<5$ per cent of the elemental sulphur abundance. Other sulphur-bearing ices are yet to be detected (Boogert, Gerakines \& Whittet 2015). Such simple sulphur-bearing volatiles are likely formed via a combination of gas-phase reactions and grain-surface chemistry. It remains unclear where the remaining sulphur is at cold, dark prestellar conditions. More recently, sulphurbearing complex organics have been detected. The S-containing methanol-analogue, $\mathrm{CH}_{3} \mathrm{SH}$, has been detected in the gas phase towards the cold core B1 (Cernicharo et al. 2012); the hot core G327.3-0.6 (Gibb et al. 2000); Orion KL (Kolesniková et al. 2014); the O-type protostar IRAS 16547-4247 (Zapata et al. 2015); Sgr B2(N2) (by Linke, Frerking \& Thaddeus 1979 and in the EMoCA survey by Müller et al. 2016); and IRAS 16293-2422 (Majumdar et al. 2016). The detection of the S-containing ethanol-analogue, $\mathrm{C}_{2} \mathrm{H}_{5} \mathrm{SH}$, has been reported towards Orion KL (Kolesniková et al. 2014), while searches towards Sgr B2(N2) remain uncertain (Müller et al. 2016). Such S-bearing complex organics can only form on the surfaces of grains.

Sulphur-bearing species have also been detected in numerous places in the Solar system and several comets. A large reservoir of sulphur is found on Jupiter's moon Io, which has an atmosphere dominated by $\mathrm{SO}_{2}$, as a result of active volcanic eruptions (e.g. Jessup, Spencer \& Yelle 2007; Moullet et al. 2008, 2013). Contrary to the ISM, the majority of cometary detections of sulphur-bearing molecules belong to $\mathrm{H}_{2} \mathrm{~S}$ and $\mathrm{S}_{2}$ (A'Hearn, Schleicher \& Feldman 1983; Mumma \& Charnley 2011). Towards the brightest comet Hale-Bopp, a greater diversity has been observed, including OCS, $\mathrm{SO}_{2}$, and $\mathrm{H}_{2} \mathrm{CS}$. The brighter comets $\mathrm{C} / 2012 \mathrm{~F} 6$ (Lemmon) and C/2014 Q2 (Lovejoy) have also been shown to contain CS (Biver et al. 2016). Currently, some of the most unique in situ data are available from the Rosetta mission on comet 67P/Churyumov-
Gerasimenko (67P/C-G hereafter; Glassmeier et al. 2007). With the Rosetta Orbiter Spectrometer for Ion and Neutral Analysis (ROSINA; Balsiger et al. 2007) aboard the orbiter, the coma has been shown to contain $\mathrm{H}_{2} \mathrm{~S}$, atomic $\mathrm{S}, \mathrm{SO}_{2}, \mathrm{SO}, \mathrm{OCS}, \mathrm{H}_{2} \mathrm{CS}, \mathrm{CS}_{2}$, and $\mathrm{S}_{2}$ (and tentatively $\mathrm{CS}$, as the mass spectrometer cannot distinguish it from $\mathrm{CO}_{2}$ ) gases (Le Roy et al. 2015). Furthermore, $\mathrm{S}_{3}$, $\mathrm{S}_{4}, \mathrm{CH}_{3} \mathrm{SH}$, and $\mathrm{C}_{2} \mathrm{H}_{6} \mathrm{~S}$ have now been detected (Calmonte et al. 2016) and information on isotopologues is available (Calmonte et al. 2017). It seems that $\sim 80$ per cent of sulphur is in refractories (dust) with only $\sim 20$ per cent hidden in volatiles (ice; see Appendix A for the details). It is likely that surface sniffing of $67 \mathrm{P} / \mathrm{C}-\mathrm{G}$ by COSAC did not reveal any sulphur-bearing species (Goesmann et al. 2015) due to a lack of mass resolution (Altwegg et al. 2017). By piecing together the sulphur puzzle from the earliest diffuse phases to the oldest cometary probes, it may be possible to disentangle the history of volatiles and refractories simultaneously, as they are formed and assembled into larger bodies.

An important parameter for the gas-phase chemistry of sulphurbearing molecules is the initial elemental $\mathrm{C} / \mathrm{O}$ ratio at the time molecules start to form, which sets the ratio between oxygen- and carbon-containing S-bearing species (e.g. as seen in the ratio between SO and CS; Watt \& Charnley 1985). Additional volatiles can be formed and/or enhanced via the passage of shocks, including $\mathrm{HS}, \mathrm{H}_{2} \mathrm{~S}, \mathrm{~S}_{2}, \mathrm{SO}^{+}$(Mitchell 1984; Pineau des Forets, Roueff \& Flower 1986; Leen \& Graff 1988; Turner 1992). Species such as $\mathrm{SO}$ and $\mathrm{SO}_{2}$ that are produced in the gas phase via reactions with $\mathrm{OH}$ upon the liberation of $\mathrm{S}$ via sputtering have become traditional shock tracers. Sulphur-bearing molecules have also been used to study discs via CS (e.g. Hasegawa et al. 1984; Blake, van Dishoeck \& Sargent 1992) and as tracers of the centrifugal barrier (disc-envelope interface) via SO (e.g. Sakai et al. 2014).

The possible formation routes in ices of species such as $S_{2}$ have been investigated by Grim \& Greenberg (1987). In their experiments, ice mixtures containing $\mathrm{H}_{2} \mathrm{~S}$ are irradiated via ultraviolet (UV) photons and the production of sulphur chains is indirectly inferred. More recently, Chen et al. (2015) have shown that energetic processing with $\mathrm{UV}$ of $\mathrm{H}_{2} \mathrm{~S}-\mathrm{CO}$ ice mixtures leads to the formation of OCS and $\mathrm{CS}_{2}$, and of $\mathrm{H}_{2} \mathrm{~S}-\mathrm{CO}_{2}$ mixtures to OCS and $\mathrm{SO}_{2}$. It is thought that $\mathrm{H}_{2} \mathrm{~S}$ forms via the hydrogenation of atomic $\mathrm{S}$ and serves as a parent species for further synthesis of sulphur-bearing ices. It is suggested that SO forms on grain surfaces via oxygen addition to $\mathrm{HS}$, and $\mathrm{SO}_{2}$ forms via oxygen addition to $\mathrm{SO}$ and/or via the association of two SO molecules. OCS can potentially form via the addition of oxygen to $\mathrm{CS}$, the addition of sulphur to $\mathrm{CO}$, and/or the association of $\mathrm{HS}$ and $\mathrm{CO}$. Experiments also predict that $\mathrm{H}_{2} \mathrm{~S}_{2}$ should be made on the grains via the association of two HS molecules; however, recent observational upper limits place it at least an order of magnitude lower in abundance than the laboratory works suggest (towards IRAS 16293-2422; Martín-Doménech et al. 2016). Neither $\mathrm{H}_{2} \mathrm{~S}_{2}$ nor $\mathrm{HS}_{2}$ have been detected with ROSINA on 67P/C-G (Calmonte et al. 2016).

This work is an attempt at piecing together the sulphur trail by comparing its budget in the warm gas on Solar system scales around a low-mass protostar to that in our own Protosolar Nebula. For this purpose, the paper will focus on the solar-analogue IRAS 16293-2422 B, as investigated by ALMA, and the best available sample of the innate Solar Nebula $-67 \mathrm{P} / \mathrm{C}-\mathrm{G}$, as unraveled by ROSINA measurements. IRAS 16293-2422 is an embedded lowmass Class 0 protostellar binary with a separation of 5.1 arcsec (or 610-750 au assuming a distance of 120-147 pc; Loinard et al. 2008; Ortiz-León et al. 2017), a combined luminosity of $21 \pm 5$ $\mathrm{L}_{\odot}$ and disc-like structures around both sources, A and B. A full 
overview of the physical and chemical properties of the source is presented in Jørgensen et al. (2016). The single dish survey with the Caltech Submillimeter Observatory (CSO) and the James Clerk Maxwell Telescope (JCMT) detected sulphur-bearing warm $\left(T_{\text {kin }} \gtrsim\right.$ $80 \mathrm{~K})$ dense $\left(\sim 10^{7} \mathrm{~cm}^{-3}\right)$ gas tracers such as $\mathrm{SO},{ }^{34} \mathrm{SO}, \mathrm{S}^{18} \mathrm{O}, \mathrm{SO}_{2}$, OCS, $\mathrm{OC}^{34} \mathrm{~S}, \mathrm{O}^{13} \mathrm{CS},{ }^{18} \mathrm{OCS}$, o- and $\mathrm{p}-\mathrm{H}_{2} \mathrm{CS}, \mathrm{H}_{2} \mathrm{C}^{34} \mathrm{~S}, \mathrm{H}_{2}^{13} \mathrm{CS}, \mathrm{SiS}$, ${ }^{29} \mathrm{SiS}$; and colder $\left(T_{\text {kin }} \sim 40 \mathrm{~K}\right)$ envelope species $\left(10^{6} \sim 10^{7} \mathrm{~cm}^{-3}\right)$ such as CS, $\mathrm{C}^{34} \mathrm{~S}, \mathrm{HCS}^{+}$, HDS (Blake et al. 1994; van Dishoeck et al. 1995 and reanalysed by Schöier et al. 2002). The single dish TIMASSS survey with IRAM-30 m and JCMT-15 m facilities expanded the list with ${ }^{34} \mathrm{SO}_{2},{ }^{13} \mathrm{CS}, \mathrm{C}^{33} \mathrm{~S}$, HDCS, and $\mathrm{C}_{2} \mathrm{~S}$ (Caux et al. 2011); and $\mathrm{CH}_{3} \mathrm{SH}$ (Majumdar et al. 2016). Interferometric observations with the Submillimeter Array (SMA) revealed the spatial distribution of $\mathrm{CS}, \mathrm{C}^{34} \mathrm{~S},{ }^{13} \mathrm{CS}, \mathrm{H}_{2} \mathrm{~S}, \mathrm{H}_{2} \mathrm{CS}, \mathrm{H}_{2} \mathrm{C}^{34} \mathrm{~S}, \mathrm{HCS}^{+}$, OCS, $\mathrm{O}^{13} \mathrm{CS}, \mathrm{SO},{ }^{33} \mathrm{SO},{ }^{34} \mathrm{SO}, \mathrm{SO}_{2},{ }^{33} \mathrm{SO}_{2},{ }^{34} \mathrm{SO}_{2}, \mathrm{SO}^{18} \mathrm{O}$, and $\mathrm{SO}^{17} \mathrm{O}$ around the binary system on scales of $\sim 190-380$ au, shedding light on the fact that source A is significantly richer in sulphur-bearing species than B (Jørgensen et al. 2011). IRAS 16293-2422 B has been targeted with ALMA in Band 9: lines of ${ }^{34} \mathrm{SO}_{2},{ }^{33} \mathrm{SO}_{2}$, and $\mathrm{SO}$ were detected in emission from a warm region near source $\mathrm{B}$; and a line of $\mathrm{H}_{2} \mathrm{~S}$ was found in absorption originating from the cold foreground gas (Baryshev et al. 2015). Based on ALMA Band 6 data at $\sim 0.6 \operatorname{arcsec} \times 0.5 \mathrm{arcsec}$ spatial resolution, Oya et al. (2016) revealed that it is possible to derive the kinematic envelope structure around source A via OCS emission, meanwhile $\mathrm{H}_{2} \mathrm{CS}$ traces both the envelope and the disc-like structure.

This paper presents the full inventory of sulphur-bearing molecules towards IRAS 16293-2422 B based on ALMA Band 7 data (Jørgensen et al. 2016). Such interferometric observations make it possible to get away from the large-scale outflow and circumbinary envelope-dominated emission and to spatially resolve the thermally desorbed molecules close to the central source. The choice to focus on source $B$ has been made, because its lines are much narrower than those observed towards source A; hence, there is less line blending. This makes it ideal for studies of isotopologues and minor species. Subsequently, ratios between various molecules are compared to those deduced for the coma gases of $67 \mathrm{P} / \mathrm{C}-\mathrm{G}$, as measured with the ROSINA instrument (Calmonte et al. 2016). Both sets of data are some of the best available for an extrasolar analogue of our Solar system and an innate Solar Nebula tracer - a comet. The differences and similarities between the two have implications for the formation history of our Solar system. Observational details are presented in Section 2 and the results are found in Section 3. Molecular ratios are computed and compared to cometary values in Section 4 and the conclusions are given in Section 5.

\section{IRAS 16293-2422 OBSERVATIONS}

This work is based on the large unbiased Protostellar Interferometric Line Survey (PILS; ${ }^{1}$ project-id: 2013.1.00278.S, PI: Jes K. Jørgensen) of IRAS 16293-2422 carried out with ALMA in the 329-363 GHz frequency range (Band 7) with a spectral resolution of $0.2 \mathrm{~km} \mathrm{~s}^{-1}$ and a beam size of $0.5 \operatorname{arcsec}$ (or 60-74 au in diameter, assuming a distance of 120-147 pc; Loinard et al. 2008; Ortiz-León et al. 2017). The data used here are continuum subtracted based on the statistical method described in Jørgensen et al. (2016). The observations are a combination of the 12- and the $7-\mathrm{m}$ dish arrays, thereby ensuring that the emission on scales up to $\sim 13$ arcsec is recovered, while also spatially resolving the target. The root-mean-square (rms) noise of the combined data set is 7-10 $\mathrm{mJy}_{\text {beam }}{ }^{-1}$ channel $^{-1}$ (or 4-5 $\mathrm{mJy}_{\text {beam }}{ }^{-1} \mathrm{~km} \mathrm{~s}^{-1}$ with beam sizes in the 0.34-0.87 range; see table 1 of Jørgensen et al. 2016). Hereafter, $\sigma=10 \mathrm{mJy}^{\text {beam }}{ }^{-1}$ channel $^{-1}$ or $5 \mathrm{mJy} \mathrm{beam}^{-1} \mathrm{~km} \mathrm{~s}^{-1}$ is adopted. Here, the data set convolved with a uniform circular restoring beam of 0.5 arcsec is used. All further details on the PILS survey, including calibration, are available in Jørgensen et al. (2016).

The spectral analysis presented in the subsequent section is carried out towards a single position of the data set - one beam $(\sim 60 \mathrm{au})$ offset from source B in the SW direction, which has also been the focal point of Coutens et al. (2016), Lykke et al. (2017), and Ligterink et al. (2017); and lies twice as far as the position studied in Jørgensen et al. (2016) in the same direction. The position lies within the high density inner regions of the disc-like structure of source B (called 'disc' for simplicity, hereafter), hence maximizing emission; while being sufficiently far from the source to avoid absorption against the strong dust continuum (Fig. 1). IRAS 162932422 is associated with one collimated pair of outflow lobes in the NW-SE direction and one less collimated in the EW direction. The studied offset point lies in the most outflow-free direction of the region to avoid any additional sources of heating. The spectrum at this position is rich in numerous narrow $\left(\sim 1 \mathrm{~km} \mathrm{~s}^{-1}\right)$ lines from various species (and at abundances a factor of 2 lower than at those seen in fig. 5 of Jørgensen et al. (2016) for the half-beam offset position in the same direction at $\sim 30$ au from the source, corresponding to the comet-forming zone). The recent analysis of complex organic emission in this direction from species such as glycolaldehyde, ethylene glycol, ethylene oxide, acetone, and propanal indicates that the temperatures at this point are $>100 \mathrm{~K}$, thus it is likely that the chosen position is probing hot inner envelope or face-on disc material heated by the protostellar B source.

\section{RESULTS AND ANALYSIS}

The one beam offset position from source B of IRAS 16293-2422 in the PILS Band 7 data set was searched for lines of all known sulphur-bearing species, including those detected in $67 \mathrm{P} / \mathrm{C}-\mathrm{G}$, and several that have been hypothesized to be present. The initial line searching and local thermal equilibrium (LTE) modelling have been carried out with CAssis. ${ }^{2}$ Thereafter, synthetic spectra have been generated with custom IDL routines. LTE is a good assumption in this case, because the densities are high at the chosen position and thus the molecules are expected to be thermalized. This assumption has also been quantified for the case of methanol $\left(\mathrm{CH}_{3} \mathrm{OH}\right)$ in section 5.1 of Jørgensen et al. (2016). It was shown that for the high densities probed by this data set $\left(\gtrsim 3 \times 10^{10} \mathrm{~cm}^{-3}\right)$, the deviation of excitation temperatures under the assumption of LTE from kinetic temperatures of non-LTE calculations is expected to be lower than 15 per cent. The explored grid of column densities corresponds to an uncertainty of $\sim 10$ per cent. Moreover, for the molecules studied in this work, all levels are well-described by a single excitation temperature.

The detected species and the best-fitting parameters, assuming a constant $T_{\mathrm{ex}}=125 \mathrm{~K}$ for all species, are tabulated in Table 1 . This value for $T_{\mathrm{ex}}$ has been determined as best-fitting by eye based on a grid of synthetic spectra with $25 \mathrm{~K}$ steps for all these species. Hence, the uncertainty on $T_{\mathrm{ex}}$ is $\sim 20$ per cent. There is no indication among this set of molecules for a different $T_{\mathrm{ex}}$. A value

\footnotetext{
${ }^{1}$ http://youngstars.nbi.dk/PILS/
}

${ }^{2}$ CASSIS has been developed by IRAP-UPS/CNRS, http://cassis.irap.omp.eu 


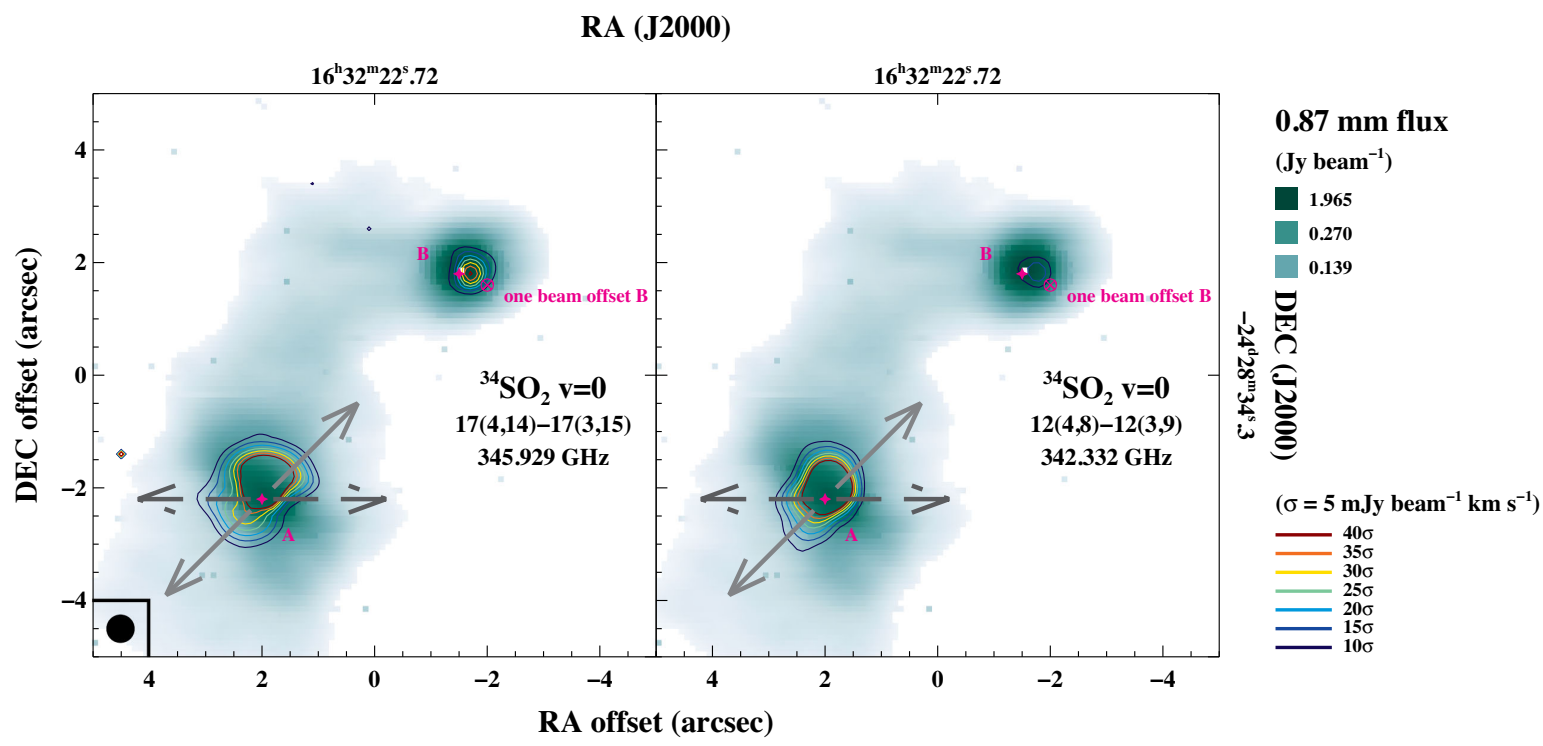

Figure 1. Integrated intensity maps of two transition of ${ }^{34} \mathrm{SO}_{2}$, see Section 3.8 for details. The centre of the pixel of the one beam offset position from IRAS $16293-2422$ B corresponds to (RA, Dec.) J2000 $=\left(16^{\mathrm{h}} 32^{\mathrm{m}} 22 .^{\mathrm{s}} 58,-24^{\circ} 28^{\prime} 32^{\prime \prime} .80\right)$. The directions of the outflows are indicated with the arrows.

Table 1. Best-fitting parameters for detected species at the one beam offset position from source B of IRAS $16293-2422^{a}$.

\begin{tabular}{|c|c|c|c|c|c|}
\hline Species & $\begin{array}{c}\text { Number of clean lines } \\
\text { (Number of lines in range) }\end{array}$ & CDMS entry & $E_{\text {up }}(\mathrm{K})$ & $N\left(\mathrm{~cm}^{-2}\right)$ & $\begin{array}{c}\text { Derived } N\left(\mathrm{~cm}^{-2}\right) \\
\text { of isotopologues }\end{array}$ \\
\hline $\mathrm{SO}_{2}, v=0$ & $13(101)$ & 64502 & $43-276$ & $1.5 \times 10^{15}$ & \\
\hline${ }^{34} \mathrm{SO}_{2}$ & $12(107)$ & 66501 & $35-185$ & $4.0 \times 10^{14}$ & $N\left(\mathrm{SO}_{2}\right)=8.8 \times 10^{15, e}$ \\
\hline $\mathrm{SO}, v=0$ & $0(8)$ & 48501 & $81-87$ & $\leq 5.0 \times 10^{14, b}$ & \\
\hline OCS, $v=0$ & $0(2)$ & 60503 & $237-254$ & $\geq 2.0 \times 10^{16, b, c}$ & \\
\hline $\mathrm{OCS}, v_{2}=1$ & $4(4)$ & 60504 & $986-1003$ & $2.0 \times 10^{17}$ & \\
\hline $\mathrm{O}^{13} \mathrm{CS}$ & $2(2)$ & 61502 & $236-253$ & $5.0 \times 10^{15}$ & $N(\mathrm{OCS})=3.5 \times 10^{17, d}$ \\
\hline $\mathrm{OC}^{34} \mathrm{~S}$ & $3(3)$ & 62505 & $231-265$ & $1.0 \times 10^{16}$ & $N(\mathrm{OCS})=2.2 \times 10^{17, e}$ \\
\hline $\mathrm{OC}^{33} \mathrm{~S}$ & $2(3)$ & 61503 & $234-268$ & $3.0 \times 10^{15}$ & $N(\mathrm{OCS})=3.8 \times 10^{17, f}$ \\
\hline${ }^{18} \mathrm{OCS}$ & $3(3)$ & 62506 & $238-272$ & $5.0 \times 10^{14}$ & $N(\mathrm{OCS})=2.8 \times 10^{17, g}$ \\
\hline $\mathrm{C}^{34} \mathrm{~S}, v=0,1$ & $1(2)$ & 46501 & 65 & $2.0 \times 10^{14}$ & $N(\mathrm{CS})=4.4 \times 10^{15, e}$ \\
\hline $\mathrm{C}^{33} \mathrm{~S}, v=0,1$ & $0(2)$ & 45502 & 65 & $8.0 \times 10^{13}$ & $N(\mathrm{CS})=1.0 \times 10^{16, f}$ \\
\hline $\mathrm{C}^{36} \mathrm{~S}$ & $1(1)$ & 48503 & 64 & $1.4 \times 10^{13}$ & $N(\mathrm{CS})=6.6 \times 10^{16, h}$ \\
\hline $\mathrm{H}_{2} \mathrm{CS}$ & $9(22)$ & 46509 & $102-419$ & $1.5 \times 10^{15}$ & \\
\hline HDCS & $6(23)$ & 47504 & $98-322$ & $1.5 \times 10^{14}$ & $N\left(\mathrm{H}_{2} \mathrm{CS}\right)=1.5 \times 10^{15}$ \\
\hline HDS & $1(10)$ & 35502 & 35 & $1.6 \times 10^{16}$ & $N\left(\mathrm{H}_{2} \mathrm{~S}\right)=1.6 \times 10^{17^{i}-18^{j}}$ \\
\hline $\mathrm{HD}^{34} \mathrm{~S}$ & $1(7)$ & 37503 & 35 & $1.0 \times 10^{15}$ & $\begin{array}{c}N(\mathrm{HDS})=2.2 \times 10^{16, e} \\
N\left(\mathrm{H}_{2} \mathrm{~S}\right)=2.2 \times 10^{17^{i}-18^{j}}\end{array}$ \\
\hline $\mathrm{CH}_{3} \mathrm{SH}, v=0-2$ & $12+(496)$ & 48510 & $127-437$ & $5.5 \times 10^{15}$ & \\
\hline
\end{tabular}

Notes. ${ }^{a}$ Assuming a source size of $0.5 \operatorname{arcsec}, \mathrm{FWHM}$ of $1 \mathrm{~km} \mathrm{~s}^{-1}$ and $T_{\mathrm{ex}}=125 \mathrm{~K}$.

${ }^{b}$ Blended.

${ }^{c}$ Optically thick.

${ }^{d}$ Assuming ${ }^{12} \mathrm{C} /{ }^{13} \mathrm{C}=69$ (Wilson 1999).

${ }^{e}$ Assuming ${ }^{32} \mathrm{~S} /{ }^{34} \mathrm{~S}=22$ (Wilson 1999).

${ }^{f}$ No estimate for the local ISM is available, so a solar ratio of ${ }^{32} \mathrm{~S} /{ }^{33} \mathrm{~S}=125$ is used (Asplund et al. 2009).

${ }^{g}$ Assuming ${ }^{16} \mathrm{O} /{ }^{18} \mathrm{O}=557$ (Wilson 1999).

${ }^{h}$ No estimate for the local ISM is available, so a solar ratio of ${ }^{32} \mathrm{~S} /{ }^{36} \mathrm{~S}=4747$ is used (Asplund et al. 2009),

${ }^{i}$ Assuming $\mathrm{D} / \mathrm{H}=0.05$, as measured with single dish observations of $\mathrm{HDS} / \mathrm{H}_{2} \mathrm{~S}$ (table 11 of van Dishoeck et al. 1995).

${ }^{j}$ Assuming $\mathrm{D} / \mathrm{H}=0.005$, as recent studies suggest that single dish observations may be overestimating deuteration as a result of underestimating optical depth.

of $125 \mathrm{~K}$ has also been derived for some complex organics (e.g. acetaldehyde, ethylene oxide, dimethyl ether and ketene; Lykke et al. 2017; Jørgensen et al. 2017); while other species show significantly higher excitation temperatures of $\sim 300 \mathrm{~K}$ (e.g. glycolaldehyde, methyl formate, formamide; Coutens et al. 2016; Jørgensen et al. 2016, 2017). These variations in excitation temperatures are likely due to different molecules tracing regions of different temperatures, which is related to the different binding energies of the species and short infall time-scales of these inner regions (Jørgensen et al. 2017). Observations at an even higher spatial resolution are necessary in order to explore this in detail. It is assumed that the source is extended and 0.5 in size. If, both, the beam and 
the source distributions are Gaussian, then the observed emission is diluted by

beam dilution $=$ source $\operatorname{size}^{2} /\left(\right.$ source size ${ }^{2}+$ beam size $\left.{ }^{2}\right)=0.5$.

A full width half-maximum (FWHM) of $1 \mathrm{~km} \mathrm{~s}^{-1}$ is taken for all species. No evidence for any deviation from this value is seen at this offset position for the sulphur-bearing molecules in this data set. The lines are spectrally resolved into about five bins. There is no indication of any additional kinematic signature such as outflows in the lines at the spectral resolution of these data at this position. The disc-like structure around source B is face-on, so Keplerian rotation is not seen. For source A, a rotating-infalling structure is seen (Pineda et al. 2012). For sources A and B, the assumed local standard of rest (LSR) velocities are 3.2 and $2.7 \mathrm{~km} \mathrm{~s}^{-1}$, respectively (Jørgensen et al. 2011). There may be a small $\left(0.1-0.2 \mathrm{~km} \mathrm{~s}^{-1}\right)$ shift in the best-fitting LSR velocities between molecules tracing somewhat warmer/cooler regions (Jørgensen et al. 2017). Subsequent subsections describe the detections on a molecule by molecule basis. Isotopic ratios for the local ISM have been taken from Wilson (1999), which are an update of Wilson \& Rood (1994), where available $\left({ }^{32} \mathrm{~S} /{ }^{34} \mathrm{~S}=22\right)$. Otherwise, solar ratios from Asplund et al. (2009) have been employed $\left({ }^{32} \mathrm{~S} /{ }^{33} \mathrm{~S}=125,{ }^{32} \mathrm{~S} /{ }^{36} \mathrm{~S}=4747\right)$. A selection of the detected lines and fitted synthetic spectra are shown in Appendix B, as supplementary online-only materials.

\section{1 $\mathrm{SO}_{2}$}

Sulphur dioxide $\left(\mathrm{SO}_{2}\right)$ in the $v=0$ state is detected based on 13 clean, non-blended lines at a column density of $1.5 \times 10^{15} \mathrm{~cm}^{-2}$. The $v_{2}=1$ state is not detected due to the covered lines having very high upper energy levels $\left(E_{u} \gtrsim 800 \mathrm{~K}\right) .{ }^{34} \mathrm{SO}_{2}$ is detected with 12 clean lines at a column density of $4.0 \times 10^{14} \mathrm{~cm}^{-2}$. For a ratio of ${ }^{32} \mathrm{~S} /{ }^{34} \mathrm{~S}=22$, the column density of $\mathrm{SO}_{2}$ should be $8.8 \times 10^{15} \mathrm{~cm}^{-2}$. This value is a factor of $\sim 6$ higher than the column density derived from the lines of $\mathrm{SO}_{2}$ itself. This may indicate that $\mathrm{SO}_{2}$ is marginally optically thick at this position; however, the synthetic fits to the data do not indicate this (a higher column density leads to an overproduction of the synthetic flux in comparison to the data). Alternatively, the sulphur isotopic ratio may differ at this position and/or for this molecule from that of the local ISM, specifically ${ }^{32} \mathrm{~S} /{ }^{34} \mathrm{~S}=3.8 \pm 0.5$ instead of 22. Finally, it may also be that the emission of $\mathrm{SO}_{2}$ stems from a non-uniform emitting area, such as optically thick clumps. Such distributed clumps would still yield a smooth (beam-diluted) emission map on large scales (such as that shown in Fig. 1). ALMA Band 9 emission maps of ${ }^{34} \mathrm{SO}_{2}$ at 0.2 arcsec resolution do indeed suggest that the emission is not homogeneously distributed within the 0.5 arcsec PILS Band 7 beam (fig. 13 of Baryshev et al. 2015). None of these possibilities can be firmly ruled out. The one beam offset position has also been searched for ${ }^{33} \mathrm{SO}_{2}, \mathrm{~S}^{18} \mathrm{OO}$, and $\mathrm{S}^{17} \mathrm{OO}$; however, all these lines are very weak, with predicted emission at the noise level of the data set.

\subsection{SO}

Sulphur monoxide (SO) in the $v=0$ state has eight lines in range. Only three are strong enough to be detected (the other five are weaker than $1 \sigma$ ) and are blended with emission from cyclopropenylidene $\left(\mathrm{c}-\mathrm{C}_{3} \mathrm{H}_{2}\right)$, methanol, ethanol $\left(\mathrm{C}_{2} \mathrm{H}_{5} \mathrm{OH}\right)$, vinyl cyanide $\left(\mathrm{C}_{2} \mathrm{H}_{3} \mathrm{CN}\right)$, glycolaldehyde $\left(\mathrm{HOCH}_{2} \mathrm{CHO}\right)$, methyl formate $(\mathrm{HCOOCH})$, and acetaldehyde $\left(\mathrm{CH}_{3} \mathrm{CHO}\right)$. Therefore, the LTE fit for this molecule is less certain and only an upper limit can be derived on its column density at a value of $5.0 \times 10^{14} \mathrm{~cm}^{-2}$. This value reflects the maximal contribution $\mathrm{SO}$ can have in the observed blended lines. In the $v=1$ state, seven lines are in range. However, all are predicted to be weaker than $1 \mathrm{mJy} \mathrm{beam}^{-1}$ and, thus, weaker than the noise in the data set. The position has also been searched for ${ }^{34} \mathrm{SO},{ }^{33} \mathrm{SO},{ }^{36} \mathrm{SO}, \mathrm{S}^{18} \mathrm{O}, \mathrm{S}^{17} \mathrm{O}$, and $\mathrm{SO}^{+}$; however, all these lines are very weak, with predicted emission below the noise level of the data set.

\subsection{OCS}

Carbonyl sulphide (OCS) in the $v=0$ state has two lines in range that are slightly blended with lines of glycolaldehyde and ethanol, respectively. The $v_{2}=1$ state is detected with four clean lines. Multiple isotopologues are also detected: $\mathrm{O}^{13} \mathrm{CS}, \mathrm{OC}^{34} \mathrm{~S}, \mathrm{OC}^{33} \mathrm{~S}$, and ${ }^{18} \mathrm{OCS}$. This is the first time $\mathrm{OC}^{33} \mathrm{~S}$ has been detected towards this source. The presence of ${ }^{33} \mathrm{~S}$ has been inferred previously via ${ }^{33} \mathrm{SO}_{2}$ (Jørgensen et al. 2012) and $\mathrm{C}^{33} \mathrm{~S}$ (Caux et al. 2011). All the other isotopologues of OCS have been detected before (Blake et al. 1994; Schöier et al. 2002; Caux et al. 2011). The data set was also searched for ${ }^{17} \mathrm{OCS}, \mathrm{OC}^{36} \mathrm{~S},{ }^{18} \mathrm{OC}^{34} \mathrm{~S},{ }^{18} \mathrm{O}^{13} \mathrm{CS}, \mathrm{O}^{13} \mathrm{C}^{34} \mathrm{~S}, \mathrm{O}^{13} \mathrm{C}^{33} \mathrm{~S}$; however, the predicted emission from these species is at the noise level of the data set.

The best-fitting column density of OCS is $2.0 \times 10^{17} \mathrm{~cm}^{-2}$, if inferred from the four lines of the $v_{2}=1$ state; and is at most $2.0 \times 10^{16} \mathrm{~cm}^{-2}$, if approximated from the two blended lines of the $v=0$ state. This immediately indicates that the $v=0$ state is optically thick and this derived column density is a lower limit. The column density of OCS can also be inferred indirectly via its optically thin isotopologues $\left(\mathrm{O}^{13} \mathrm{CS}, \mathrm{OC}^{34} \mathrm{~S}\right.$, and $\left.{ }^{18} \mathrm{OCS}\right)$ and the respective local ISM isotopic ratios. The derived numbers are in Table 1 and are within a factor of 2 from the column density calculated from the $v_{2}=1$ state. They are an order of magnitude higher than that based on the $v=0$ state confirming its optical thickness. The average of these four optically thin estimates is $(2.6 \pm 0.9) \times 10^{17} \mathrm{~cm}^{-2}$. The average of the values based on just the three isotopologues is $(2.8 \pm 0.7) \times 10^{17} \mathrm{~cm}^{-2}$ and is the best estimate of the column density of OCS at this position. As an additional check, the solar ratio of ${ }^{32} \mathrm{~S} /{ }^{33} \mathrm{~S}=125$ can be used to derive the column density of OCS from its $\mathrm{OC}^{33} \mathrm{~S}$ isotopologue, since no estimate for the local ISM is available. This yields a value of $3.8 \times 10^{17} \mathrm{~cm}^{-2}$, which is consistent within a factor of 1.4 with the average value derived from three other isotopologues. Alternatively, the observed column density of $\mathrm{OC}^{33} \mathrm{~S}$ and the estimated average column density of OCS can be used to calculate ${ }^{32} \mathrm{~S} /{ }^{33} \mathrm{~S}$ for IRAS $16293-2422 \mathrm{~B}$ to be $93 \pm 11$ (with $1 \sigma$ significance), which is marginally sub-solar compared with the solar value of 125 at the highest end of the error bars.

\section{$3.4 \mathrm{CS}$}

Carbon monosulphide (CS) in all the $v=0,1,2,3,4$ vibrational states has five rotational lines in range. Only one of them $(v=0$, $J=7-6)$ is expected to be strong enough to be detected $\left(E_{u}=66 \mathrm{~K}\right.$ and $\left.A_{i j}=8.40 \times 10^{-4} \mathrm{~s}^{-1}\right)$, but is blended with ethyl cyanide $\left(\mathrm{C}_{2} \mathrm{H}_{5} \mathrm{CN}\right)$ and ethylene glycol $\left(\left(\mathrm{CH}_{2} \mathrm{OH}\right)_{2}\right)$. The $\mathrm{C}^{34} \mathrm{~S}$ isotopologue in the $v=0,1$ state has 2 lines in range. One is cleanly detected, while the second is predicted to be too weak for a detection due to a high value of $E_{u} \sim 1880 \mathrm{~K}$. The best-fitting column density is $2.0 \times 10^{14} \mathrm{~cm}^{-2}$. The $\mathrm{C}^{33} \mathrm{~S}$ isotopologue in the $v=0,1$ also has two lines in range. One is cleanly detected, while the second 
Table 2. Upper limits $(1 \sigma)$ on the column densities of selected species at the one beam offset position from source B of IRAS $16293-2422^{a}$.

\begin{tabular}{|c|c|c|c|}
\hline Species & Catalogue and entry number & Number of lines in range & $N\left(\mathrm{~cm}^{-2}\right)$ \\
\hline gauche- $\mathrm{C}_{2} \mathrm{H}_{5} \mathrm{SH}$ & CDMS 62523 & 1329 & $\leq 3.0 \times 10^{15}$ \\
\hline anti- $\mathrm{C}_{2} \mathrm{H}_{5} \mathrm{SH}$ & CDMS 62524 & 470 & $\leq 6.0 \times 10^{14}$ \\
\hline gauche- $\mathrm{C}_{2} \mathrm{H}_{5}^{34} \mathrm{SH}$ & CDMS 64517 & 1106 & $\leq 1.0 \times 10^{15}$ \\
\hline $\mathrm{S}_{2}$ & JPL 64001 & 1 & $\lesssim 2.2 \times 10^{16, k}$ \\
\hline $\mathrm{S}_{3}$ & JPL 96002 & 303 & $\leq 4.0 \times 10^{15}$ \\
\hline $\mathrm{S}_{4}$ & JPL 128001 & 952 & $\leq 1.0 \times 10^{16}$ \\
\hline $\mathrm{HS}_{2}$ & CDMS 65509 & 227 & $\leq 5.0 \times 10^{14}$ \\
\hline $\mathrm{H}_{2} \mathrm{~S}_{2}$ & CDMS 66507 & 33 & $\leq 9.0 \times 10^{14}$ \\
\hline $\mathrm{S}_{2} \mathrm{O}, v=0$ & CDMS 80503 & 378 & $\leq 1.0 \times 10^{15}$ \\
\hline cis- $\mathrm{S}_{2} \mathrm{O}_{2}$ & CDMS 96501 & 567 & $\leq 1.0 \times 10^{14}$ \\
\hline $\mathrm{HCS}$ & CDMS 45507 & 6 & $\leq 1.0 \times 10^{15}$ \\
\hline HSC & CDMS 45508 & 29 & $\leq 1.0 \times 10^{14}$ \\
\hline $\mathrm{HCS}^{+}$ & CDMS 45506 & 1 & $\lesssim 2.5 \times 10^{13}$ \\
\hline $\mathrm{DCS}^{+}$ & CDMS 46505 & 1 & $\lesssim 5.0 \times 10^{12}$ \\
\hline $\mathrm{HC}^{34} \mathrm{~S}^{+}$ & CDMS 47502 & 1 & $\approx 6.0 \times 10^{12}$ \\
\hline $\mathrm{H}_{2} \mathrm{C}^{34} \mathrm{~S}$ & CDMS 48508 & 20 & $\leq 7.0 \times 10^{13}$ \\
\hline $\mathrm{D}_{2} \mathrm{CS}$ & CDMS 48507 & 32 & $\leq 1.0 \times 10^{14}$ \\
\hline $\mathrm{CCS}$ & CDMS 56502 & 14 & $\leq 4.0 \times 10^{13}$ \\
\hline $\mathrm{H}_{2} \mathrm{C}_{2} \mathrm{~S}$ & CDMS 58501 & 63 & $\leq 5.0 \times 10^{14}$ \\
\hline c- $\mathrm{C}_{2} \mathrm{H}_{4} \mathrm{~S}$ & CDMS 60509 & 191 & $\leq 1.0 \times 10^{14}$ \\
\hline $\mathrm{NS}, v=0$ & CDMS 46515 & 20 & $\leq 2.0 \times 10^{13}$ \\
\hline NCS & CDMS 58504 & 12 & $\leq 4.0 \times 10^{14}$ \\
\hline HNCS, a-type ${ }^{k}$ & CDMS 59503 & 10 & $\leq 1.0 \times 10^{14}$ \\
\hline HSCN & CDMS 59505 & 75 & $\leq 1.0 \times 10^{14}$ \\
\hline
\end{tabular}

Notes. ${ }^{a}$ Not possible to derive an accurate upper limit based on one blended line, so the value from Martín-Doménech et al. (2016) is adopted, ignoring the difference in beam sizes.

${ }^{k} \mathrm{~B}$-type (CDMS entry 59504) has two lines in range, which cannot be detected due to very high $E_{\text {up }} \sim 1700 \mathrm{~K}$.

is again too weak to detect as $E_{u} \sim 1887 \mathrm{~K}$. The best-fitting column density is $8.0 \times 10^{13} \mathrm{~cm}^{-2}$. The fourth S-isotopologue $\mathrm{C}^{36} \mathrm{~S}$ has one line in range; and it is cleanly detected $\left(E_{u}=64 \mathrm{~K}\right.$ and $\left.A_{i j}=7.66 \times 10^{-4} \mathrm{~s}^{-1}\right)$, giving a best-fitting column density of $1.4 \times 10^{13} \mathrm{~cm}^{-2}$. This is a tentative first-time detection of ${ }^{36} \mathrm{~S}$ towards a low-mass protostar. $\mathrm{C}^{36} \mathrm{~S}$ has been previously detected towards high-mass hot cores (Mauersberger et al. 1996) as a first-time detection of interstellar ${ }^{36} \mathrm{~S}$. More lines are required to confirm this detection; however, the isotopic ratios are in agreement with solar ratios. Other excitation states and isotopologues lack lines in the observed frequency range, specifically: CS in the $v=1-0,2-1$ and $v=2-0$ states; $\mathrm{C}^{34} \mathrm{~S}$ in the $v=1-0$ state; ${ }^{13} \mathrm{CS}$ in the $v=0,1$ and $v=1-0$ states; ${ }^{13} \mathrm{C}^{34} \mathrm{~S}$ and ${ }^{13} \mathrm{C}^{33} \mathrm{~S}$. Meanwhile, ${ }^{13} \mathrm{C}^{36} \mathrm{~S}$ has one line in range, but it is a clear non-detection. $\mathrm{CS}^{+}$has two lines in range; however, one is too weak $\left(A_{i j}=7.12 \times 10^{-7} \mathrm{~s}^{-1}\right)$, while the other suffers from blending and absorption.

Assuming the local ISM ratio of ${ }^{32} \mathrm{~S} /{ }^{34} \mathrm{~S}=22$, the column density of CS can be estimated at $4.4 \times 10^{15} \mathrm{~cm}^{-2}$ from its $\mathrm{C}^{34} \mathrm{~S}$ isotopologue. If this column density is used to fit the detected line of CS suffering from absorption, then the synthetic line width matches the observed. Thus, this is the best available estimate of the column density of CS. Based on $\mathrm{C}^{33} \mathrm{~S}$ and $\mathrm{C}^{36} \mathrm{~S}$, the column density of $\mathrm{CS}$ can be derived to be $1.0 \times 10^{16} \mathrm{~cm}^{-2}$ and $6.6 \times 10^{16} \mathrm{~cm}^{-2}$, respectively, which is factors of 2.3 and 15 larger than the value derived based on $\mathrm{C}^{34} \mathrm{~S}$. This may imply that either the $\mathrm{C}^{36} \mathrm{~S}$ column density is poorly constrained by the single line; or that the solar isotopic ratio is not applicable for IRAS 16293-2422 B. Alternatively, the observed column densities of $C^{33} S$ and $C^{36} S$ in conjunction with the estimated CS column density can be used to calculate ${ }^{32} \mathrm{~S} /{ }^{33} \mathrm{~S}$ and ${ }^{32} \mathrm{~S} /{ }^{36} \mathrm{~S}$ for IRAS $16293-2422 \mathrm{~B}$ to be $55 \pm 8$ and $314 \pm 44$ (both with $1 \sigma$ significance), i.e. a factor of 2 and an order of magnitude lower than the solar values of 125 and 4747, respectively.

\section{5 $\mathrm{H}_{2} \mathrm{CS}$}

Thioformaldehyde $\left(\mathrm{H}_{2} \mathrm{CS}\right)$ is detected with 9 clean lines at a column density of $1.5 \times 10^{15} \mathrm{~cm}^{-2} \cdot \mathrm{H}_{2} \mathrm{C}^{34} \mathrm{~S}$ cannot be cleanly detected due to a lack of non-blended lines, thus only an upper limit of $7.0 \times 10^{13} \mathrm{~cm}^{-2}$ can be derived (Table 2). The tentative assignments of the strongest lines of $\mathrm{H}_{2} \mathrm{C}^{34} \mathrm{~S}$ at this maximal column density would imply a column density of $1.5 \times 10^{15} \mathrm{~cm}^{-2}$ for $\mathrm{H}_{2} \mathrm{CS}$ (assuming ${ }^{32} \mathrm{~S} /{ }^{34} \mathrm{~S}=22$ ), thus testifying to $\mathrm{H}_{2} \mathrm{CS}$ emission being (very close to) optically thin.

HDCS is detected with six clean lines at a column density of $1.5 \times 10^{14} \mathrm{~cm}^{-2}$. Since $\mathrm{H}_{2} \mathrm{CS}$ appears to be optically thin, the bestfitting column densities can be used to calculate the $\mathrm{HDCS} / \mathrm{H}_{2} \mathrm{CS}$ ratio to be $0.1 \pm 0.014$ and $\mathrm{a} \mathrm{D} / \mathrm{H}=0.05 \pm 0.007$. $^{3}$ A lower level of deuteration is ruled out based on the low quantity of $\mathrm{H}_{2} \mathrm{C}^{34} \mathrm{~S}$. This implies that thiofomaldehyde is highly singly deuterated (5 per cent), but comparable to many oxygen-bearing complex organic species (Jørgensen et al. 2017).

$\mathrm{D}_{2} \mathrm{CS}$ is not detected with an upper limit of $1.0 \times 10^{14} \mathrm{~cm}^{-2}$ (Table 2). This yields a $\mathrm{D}_{2} \mathrm{CS} / \mathrm{HDCS}$ ratio of $<0.67$, and a $\mathrm{D}_{2} \mathrm{CS} / \mathrm{H}_{2} \mathrm{CS}$ ratio of $<0.067$. Persson et al. (2018) derived the ratios between the deuterated isotopologues of formaldehyde $\left(\mathrm{H}_{2} \mathrm{CO}\right)$ to be $\mathrm{HDCO} / \mathrm{H}_{2} \mathrm{CO}=0.065 \pm 0.01, \mathrm{D}_{2} \mathrm{CO} / \mathrm{HDCO}=0.128_{-0.041}^{+0.033}$, and

\footnotetext{
${ }^{3}$ A correction for the statistical factor of 2 is applied, which assumes that deuteration events are mutually exclusive
} 
$\mathrm{D}_{2} \mathrm{CO} / \mathrm{H}_{2} \mathrm{CO}=0.0064 \pm 0.001$. This means that in comparison to formaldehyde, the sulphur-bearing analogue (thioformaldehyde) is a factor of $\sim 1.5$ more singly deuterated relative to its main isotopologue, at most a factor of $\sim 5$ more doubly deuterated relative to its singly deuterated isotopologue and at most a factor of $\sim 10$ more doubly deuterated relative to its main isotopologue. This may suggest that thiofomaldehyde forms under even more deuterium-rich conditions than formaldehyde or that it undergoes fewer chemical reactions that would lower its level of deuteration from the time of initial synthesis. Other isotopologues have not been detected: $\mathrm{H}_{2} \mathrm{C}^{33} \mathrm{~S}$ and $\mathrm{H}_{2}^{13} \mathrm{CS}$. Higher sensitivity data would be useful for ascertaining the column density of $\mathrm{D}_{2} \mathrm{CS}$ and firmly verifying the degree of deuteration of thioformaldehyde.

\section{6 $\mathrm{H}_{2} \mathrm{~S}$}

Hydrogen sulphide $\left(\mathrm{H}_{2} \mathrm{~S}\right)$ has one line in the observed frequency range; however, it is not detected due to a high upper energy level $\left(E_{u}=758 \mathrm{~K}\right)$ and line weakness $\left(A_{i j}=6.28 \times 10^{-9} \mathrm{~s}^{-1}\right)$. On the other hand, its isotopologue HDS has 10 lines in range. One of those is a clear detection at the $65 \sigma$ level, while the others have upper energy levels that are too high $\left(E_{u}>537 \mathrm{~K}\right)$ and thus are not expected to be strong. The best-fitting column density is $1.6 \times 10^{16} \mathrm{~cm}^{-2}$. $\mathrm{HD}^{34} \mathrm{~S}$ has seven lines in range. One is strong-enough and blendfree to be detected, while all others have upper energy levels that are too high $\left(E_{u}>536 \mathrm{~K}\right)$ to be stronger than the noise level. The bestfitting column density is $1.0 \times 10^{15} \mathrm{~cm}^{-2}$. Assuming that ${ }^{32} \mathrm{~S} /{ }^{34} \mathrm{~S}=$ 22 gives a column density of $2.2 \times 10^{16} \mathrm{~cm}^{-2}$ for HDS, which closely (a factor of 1.4) agrees with the value derived based on its single observed line. Other isotopologues are not detected. $\mathrm{H}_{2}^{34} \mathrm{~S}$ and $\mathrm{H}_{2}^{33} \mathrm{~S}$ do not have any lines in the observed frequency range. $\mathrm{D}_{2} \mathrm{~S}$ has four lines in range; however, only two are above the noise level and both suffer from blending, thus no clear detection is possible. $\mathrm{D}_{2}^{34} \mathrm{~S}$ has two lines in range, but one is blended with a strong absorption feature; and the other with emission lines of acetaldehyde and the ${ }^{13} \mathrm{C}$-isotopologue of ethyl cyanide $\left(\mathrm{C}_{2} \mathrm{H}_{5}^{13} \mathrm{CN}\right)$; thus, no confident detection can be claimed.

Assuming $\mathrm{D} / \mathrm{H}=0.05$, as measured with single dish observations of $\mathrm{HDS} / \mathrm{H}_{2} \mathrm{~S}$ (table 11 of van Dishoeck et al. $1995^{4}$ ), the column density of $\mathrm{H}_{2} \mathrm{~S}$ can be estimated from HDS and $\mathrm{HD}^{34} \mathrm{~S}$ (Table 1). The average of these two values of $(1.9 \pm 0.3) \times 10^{17} \mathrm{~cm}^{-2}$ is the best estimate of the column density of $\mathrm{H}_{2} \mathrm{~S}$ at this position. It is possible that single dish observations may be overestimating deuteration, either because of sampling colder material or as a result of underestimating optical thickness of the main species. The lowest $\mathrm{D} / \mathrm{H}$ ratio seen towards IRAS $16293-2422$ is 0.01 ( 1 per cent) for HNCO (Coutens et al. 2016). To account for this other extreme for the case of $\mathrm{H}_{2} \mathrm{~S}$, a D/H $=0.005$ may be assumed, increasing the best estimate to be $(1.9 \pm 0.3) \times 10^{18} \mathrm{~cm}^{-2}$.

The previously obtained SMA observations of IRAS 16293-2422 have detected a line of $\mathrm{H}_{2} \mathrm{~S}$ around $\sim 216.71 \mathrm{GHz}$ (see fig. 6 of Jørgensen et al. 2011). LTE modelling assuming a beam size of 3 arcsec, a spectral resolution of $0.56 \mathrm{~km} \mathrm{~s}^{-1}$ (as given in table 1 of Jørgensen et al. 2011), FWHM of $1 \mathrm{~km} \mathrm{~s}^{-1}$ and a source size of 0.5 arcsec (as assumed for the ALMA observations) shows that the line is optically thick. In order to match the observed line intensity of $\sim 2 \mathrm{Jy} \mathrm{beam}^{-1}$, a larger source size is necessary. Upon the assumption of a source 1 arcsec in size, the lower limit on the column density of $\mathrm{H}_{2} \mathrm{~S}$ is $4.0 \times 10^{16} \mathrm{~cm}^{-2}$. This illustrates the uncertainty in the emitting area of this molecule. Alternatively, a non-detection of the sole line in range of the SMA observations of $\mathrm{H}_{2}^{34} \mathrm{~S}$ at $\sim 226.70 \mathrm{GHz}$ does not yield a strongly constraining column density estimate. Assuming the same parameters, in order to be weaker than the SMA $3 \sigma$ noise level (where $\sigma=0.24 \mathrm{Jy} \mathrm{beam}^{-1}$ channel $^{-1}$; table 1 of Jørgensen et al. 2011), the column density of $\mathrm{H}_{2}^{34} \mathrm{~S}$ must not exceed $4.0 \times 10^{19} \mathrm{~cm}^{-2}$. Assuming ${ }^{32} \mathrm{~S} /{ }^{34} \mathrm{~S}=22$, the upper limit on the column density of $\mathrm{H}_{2} \mathrm{~S}$ is $8.8 \times 10^{20} \mathrm{~cm}^{-2}$. This is consistent with the limits obtained with the uncertain limit obtained from HDS. Dedicated high-resolution ALMA observations are needed in order to constrain the spatial distribution and column density of $\mathrm{H}_{2} \mathrm{~S}$.

\section{7 $\mathrm{CH}_{3} \mathrm{SH}$ and other species}

Methyl mercaptan $\left(\mathrm{CH}_{3} \mathrm{SH}\right.$, also known as methanethiol) in the $v=0-2$ state is detected with more than 12 clean lines at a column density of $5.5 \times 10^{15} \mathrm{~cm}^{-2}$, which is comparable to the column density of $\mathrm{H}_{2} \mathrm{CS}$. Thioformaldehyde is a likely precursor for methyl mercaptan's grain-surface formation pathways, analogously to formaldehyde being a precursor for methanol.

No other sulphur-bearing species were detected towards the one beam offset position; however, selected $1 \sigma$ upper limits have been derived and are given in Table 2. The values correspond to the maximal column densities at the fixed $T_{\mathrm{ex}}=125 \mathrm{~K}$ that the species can have in order to not exceed the observed flux at the frequencies of their emission lines. The obtained upper limits for all three versions of ethyl mercaptan $\left(\mathrm{C}_{2} \mathrm{H}_{5} \mathrm{SH}\right)$ are lower than the observed column density of methyl mercaptan by at most an order of magnitude, which is consistent with it being a step-up in chemical complexity.

The derived upper limits for the column densities of $\mathrm{HS}_{2}$ and $\mathrm{H}_{2} \mathrm{~S}_{2}$ are more constraining than those obtained by Martín-Doménech et al. (2016) owing to the much smaller beam size of the PILS Band 7 data. It is not possible to obtain an accurate upper limit for $\mathrm{S}_{2}$ as only one line is covered in this data set; however, the upper limit derived by Martín-Doménech et al. (2016) in a much larger beam size based on two lines is consistent with the observed nondetection. Carbon-sulphur chains larger than $\mathrm{CS}$, e.g. $\mathrm{C}_{2} \mathrm{~S}$, are not detected.

Upper limits on other likely carriers of sulphur in conjunction with $\mathrm{H}, \mathrm{C}, \mathrm{O}$, and $\mathrm{N}$ atoms are low (almost all $\lesssim 10^{15} \mathrm{~cm}^{-2}$, compared with $\gtrsim 10^{17} \mathrm{~cm}^{-2}$ for OCS and $\mathrm{H}_{2} \mathrm{~S}$ ), which indicates that the dominating volatile sulphur reservoirs have been accounted for. The only remaining untapped sulphur reservoir would be the refractory/dust component. All other species that were not detected at this position are listed in Appendix D.

\subsection{Integrated intensity maps}

In order to gain insight into the spatial distribution of the emission of sulphur-bearing species, integrated intensity maps of two ${ }^{34} \mathrm{SO}_{2}$, $v=0$ transitions (at 342.332 and $345.929 \mathrm{GHz}$ ) are displayed in Fig. 1. This molecule has been chosen as it is firmly detected with many lines; and its emission is optically thin, without a doubt, at the one beam offset position from IRAS 16293-2422 B. The pixels in the maps are 0.1 arcsec $\times 0.1$ arcsec in order to comfortably oversample the spatial resolution of $0.5 \mathrm{arcsec}$ of the synthesized beam. The integration is performed over nine spectral bins (one containing the line frequency and four to each side of that bin). Hence, the integrated maps cover $\pm 0.9 \mathrm{~km} \mathrm{~s}^{-1}$ around the source velocity of $\mathrm{B}$ of $2.7 \mathrm{~km} \mathrm{~s}^{-1}$ (i.e. $v_{\mathrm{lsr}}$ from +1.9 to $+3.6 \mathrm{~km} \mathrm{~s}^{-1}$ at the spectral resolution of the data of $0.244 \mathrm{MHz}$ or $0.2 \mathrm{~km} \mathrm{~s}^{-1}$ ). This implies that the maps are appropriate for the emission stemming from quiescent gas near source B; however, near source A they are less representative even though the systemic velocity of $3.2 \mathrm{~km} \mathrm{~s}^{-1}$ of source A is covered. All emission lines near source A are likely wider than 
Table 3. Molecular ratios relative to $\mathrm{H}_{2} \mathrm{~S}$ and OCS as measured with PILS interferometric ALMA observations at the one beam offset position from source B of IRAS 16293-2422 and with previous single dish (SD) work $^{m}$.

\begin{tabular}{|c|c|c|c|c|c|c|c|}
\hline \multirow[t]{2}{*}{ Species } & \multirow[t]{2}{*}{$N\left(\mathrm{~cm}^{-2}\right)$} & \multicolumn{2}{|c|}{$\begin{array}{l}\text { Molecular ratios relative } \\
\text { to } \mathrm{H}_{2} \mathrm{~S} \text { (per cent) }\end{array}$} & \multicolumn{4}{|c|}{ Molecular ratios relative to OCS (per cent) } \\
\hline & & ALMA B & SD constant & ALMA B & SD constant & SD inner & SD outer \\
\hline OCS & $2.8 \times 10^{17}$ & $147-15$ & 438 & 100 & 100 & 100 & 100 \\
\hline SO & $5.0 \times 10^{14}$ & $0.3-0.03$ & 275 & 0.2 & 63 & 100 & 117 \\
\hline $\mathrm{SO}_{2}$ & $1.5 \times 10^{15}$ & $0.8-0.08$ & 39 & 0.5 & 9 & 40 & 15 \\
\hline
\end{tabular}

Note. ${ }^{m}$ Tables 5 and 6 of Schöier et al. (2002).

$2 \mathrm{~km} \mathrm{~s}^{-1}$; thus, some of the flux from the line wings is missed when integrating over just nine bins. Moreover, there is a steep velocity gradient around source A. Integration over a wider velocity range will pollute the maps with emission from other species. In fact, even with integration over this velocity range already leads to contamination by emission from broadened neighbouring lines. Bearing in mind these limitations, Fig. 1 clearly shows that source A has stronger emission in ${ }^{34} \mathrm{SO}_{2}$ and also on larger scales. The $346 \mathrm{GHz}$ line shows a higher flux than the $342 \mathrm{GHz}$ line around source B, while little difference is seen around source A. Since emission in two lines from the same molecule differs spatially, the physical conditions exciting the ${ }^{34} \mathrm{SO}_{2}$ molecule must also differ around the two sources.

These maps show convincingly that the ${ }^{34} \mathrm{SO}_{2}$ emission near source B is dominated by material on small scales either from the disc or the inner envelope (barely no difference is seen when analysing the 12-m data instead of the combination with ACA). For the $342 \mathrm{GHz}$ line of ${ }^{34} \mathrm{SO}_{2}$, the line peaks at $\sim 38 \mathrm{mJy}_{\text {beam }}{ }^{-1}$. Integrating over nine spectral bins yields the total flux of this line at the one beam offset position of $\sim 26 \mathrm{mJy}_{\text {beam }}{ }^{-1} \mathrm{~km} \mathrm{~s}^{-1}$. The total flux of this line integrated over the entirety of source B (Fig. 1) is $\sim 150 \mathrm{mJy} \mathrm{km} \mathrm{s}^{-1}$ (for RA $\in[-1.0 \operatorname{arcsec},-2.4 \operatorname{arcsec}]$ and Dec. $\in[1.3 \operatorname{arcsec}, 2.2 \operatorname{arcsec}])$. Taking the ratio of these two numbers implies that $\sim 17$ per cent of the total $342 \mathrm{GHz}$ line emission of ${ }^{34} \mathrm{SO}_{2}$ towards source $\mathrm{B}$ is picked up in a beam solid angle at the one beam offset position. For the purposes of this paper, which are to compare and contrast the chemical composition of the smallest disc scales around source B to those of comet $67 \mathrm{P} / \mathrm{C}-\mathrm{G}$, it has been chosen to focus on this single position rather than the entire emitting area (which also differs per molecule). Emission from other species, such as CS and its isotopologues, is likely a superposition of extended (envelope) and the more compact (disc) components. However, these ALMA observations are marginally sensitive to envelope emission, since any structure that is smooth on scales of more than $\sim 13$ arcsec is filtered, even by the ACA.

\section{DISCUSSION}

\subsection{Comparison with single dish observations}

The presented interferometric observations spatially resolve the hot inner regions near the source B continuum peak, meaning that column densities can be derived and compared on the same scales unambiguously. Moreover, it is possible to detect weaker lines from multiple isotopologues, thereby allowing a better determination of the optical depth. By comparing molecular ratios of interferometric and single dish observations, it is possible to disentangle whether molecules primarily emit on large scales or small inner scales near the source, or whether they are associated with outflows. For this purpose, Table 3 has been compiled with molecular ratios of the detected species relative to $\mathrm{H}_{2} \mathrm{~S}$ and OCS (where available), as obtained with the PILS survey with ALMA at the one beam offset position and as compiled from previous single dish observations of Schöier et al. (2002). Since single dish observations cannot spatially resolve the emission, three different sets of abundances are typically provided: one assuming a constant abundance profile and one assuming a jump abundance profile, giving an inner (hot) abundance and an outer (cold) one.

Under the assumption of a constant abundance profile, relative to $\mathrm{H}_{2} \mathrm{~S}$, the interferometric ratios are lower than those from single dish observations: by a factor of 3-30 for OCS, by 3-4 orders of magnitude for $\mathrm{SO}$, and by 2-3 orders of magnitude for $\mathrm{SO}_{2}, \mathrm{CS}$, and $\mathrm{H}_{2} \mathrm{CS}$ (Table 3). Relative to OCS, the interferometric ratio is a factor of 3-30 higher than the single dish value for $\mathrm{H}_{2} \mathrm{~S}$. All other interferometric ratios remain lower than the single dish: by 2 orders of magnitude for $\mathrm{SO}$, by a factor of $\sim 18$ for $\mathrm{SO}_{2}$, by a factor of $\sim 27$ for $\mathrm{CS}$ and by a factor of $\sim 6$ for $\mathrm{H}_{2} \mathrm{CS}$. Under the assumption of a jump abundance profile, relative to OCS, the differences are either comparable or exacerbated further. The inner ratio is expected to yield the most meaningful comparison with interferometric data, as it is the closest estimate for the hot inner regions studied in this work.

The derived lower interferometric ratios mean that sulphurbearing species emit on small and large scales. Single dish observations are, therefore, a combination of beam-diluted disc-scale emission (isotopologues detected) and large-scales emission from the envelope and outflows. The largest differences are seen for SO, which is a well-known shock tracer that shows larger line widths in the single dish data (Blake et al. 1994). Some $\mathrm{SO}_{2}$ emission likely originates from outflows as well. Meanwhile, OCS, $\mathrm{H}_{2} \mathrm{~S}$ and $\mathrm{H}_{2} \mathrm{CS}$ emit also from the envelope. In fact, $\mathrm{H}_{2} \mathrm{CS}$ is one of the few species detected at large distances from source $\mathrm{A}$ (appendices $\mathrm{C} 1$ and $\mathrm{C} 2$ of Murillo et al. 2017), definitively pointing to its origins in the extended envelope. However, the detection of all these molecules with interferometric data testifies to their presence on small scales of the disc and/or inner envelope as well. Unfortunately, it is not meaningful to quantify these differences, since single dish observations also encompass source A. The emission from sulphur-bearing species is seen to be brighter towards source A, which may correlate with A powering stronger outflows. It is beyond the scope of this work to quantify the individual contributions of the two sources in single dish observations.

\subsection{Comparison with interstellar ices}

The interstellar ices in clouds and cores prior to the onset of star formation (i.e. prestellar ices) can only be observed in 
Table 4. Molecular ratios relative to $\mathrm{H}_{2} \mathrm{~S}$ and OCS as measured with these interferometric ALMA observations at the one beam offset position from source B of IRAS $16293-2422$ in comparison to those of $67 \mathrm{P} / \mathrm{C}-\mathrm{G}^{n}$ and ISM ices towards W33 $\mathrm{A}^{\circ}$.

\begin{tabular}{|c|c|c|c|c|c|c|}
\hline \multirow[t]{2}{*}{ Species } & \multirow[t]{2}{*}{$N\left(\mathrm{~cm}^{-2}\right)$} & \multicolumn{2}{|c|}{$\begin{array}{l}\text { Molecular ratios relative } \\
\text { to } \mathrm{H}_{2} \mathrm{~S} \text { (per cent) }\end{array}$} & \multicolumn{3}{|c|}{ Molecular ratios relative to OCS (per cent) } \\
\hline & & ALMA B & $67 \mathrm{P} / \mathrm{C}-\mathrm{G}$ & ALMA B & $67 \mathrm{P} / \mathrm{C}-\mathrm{G}$ & ISM ices W33A \\
\hline OCS & $2.8 \times 10^{17}$ & $147-15$ & $4.43 \pm 0.15$ & 100 & 100 & 100 \\
\hline SO & $5.0 \times 10^{14}$ & $0.3-0.03$ & $7.06 \pm 0.17$ & 0.2 & 159 & - \\
\hline $\mathrm{SO}_{2}$ & $1.5 \times 10^{15}$ & $0.8-0.08$ & $12.5 \pm 0.3$ & 0.5 & 282 & $3-7$ \\
\hline $\mathrm{S}_{2}$ & $\leq 2.2 \times 10^{16}$ & $\leq 12$ & $0.25 \pm 0.05$ & $\leq 8$ & 6 & - \\
\hline $\mathrm{HS}_{2}$ & $\leq 5.0 \times 10^{14}$ & $\leq 0.3$ & $\leq 0.01$ & $\leq 0.2$ & $\leq 0.2$ & - \\
\hline $\mathrm{H}_{2} \mathrm{~S}_{2}$ & $\leq 9.0 \times 10^{14}$ & $\leq 0.5$ & $\leq 0.057$ & $\leq 0.3$ & $\leq 1$ & - \\
\hline $\mathrm{H}_{2} \mathrm{CS}$ & $1.5 \times 10^{15}$ & 0.8 & $2.25 \pm 1.38$ & 0.5 & 51 & - \\
\hline $\mathrm{CH}_{3} \mathrm{SH}$ & $5.5 \times 10^{15}$ & $3-0.3$ & $3.5 \pm 1.0$ & 2 & 79 & - \\
\hline $\mathrm{C}_{2} \mathrm{H}_{5} \mathrm{SH}$ & $\leq 1.8 \times 10^{15, p}$ & $\leq 0.9$ & $0.0338 \pm 0.018$ & $\leq 0.6$ & 0.08 & - \\
\hline
\end{tabular}

Notes. ${ }^{n}$ See Section 4.3 for the details on the ROSINA data.

${ }^{o}$ Ice ratios in the cold outer protostellar envelope of the high-mass protostar W33A, which are assumed to be representative of ISM ices (Boogert et al. 1997; van der Tak et al. 2003).

${ }^{p}$ Using the average of the upper limits on the column densities of gauche- $\mathrm{C}_{2} \mathrm{H}_{5} \mathrm{SH}$ and anti- $\mathrm{C}_{2} \mathrm{H}_{5} \mathrm{SH}$.

absorption against a bright background source. Observations towards the high-mass protostar W33A also show deep absorption features corresponding to ices in a protostellar envelope, which are water-dominated, but also contain trace species, including $\mathrm{CH}_{3} \mathrm{OH}$ (Boogert et al. 1997; van der Tak et al. 2003). The ices in the outer cold protostellar envelope have been suggested to be chemically representative of prestellar ices (just prior to star formation) based on observations of non-sulphur bearing molecules (Boogert et al. 2015). Table 4 contains the ice molecular ratios relative to OCS as observed towards W33A in its outer cold protostellar envelope and are assumed to be representative of ISM ices, thus called as such. It is interesting to compare ISM ices to the warm (>100 K) gas near the forming B protostar of IRAS 16293-2422 to assess the degree of processing between the cold outer protostellar envelope ices and this earliest warm embedded protostellar phase, assuming that the ices have just thermally desorbed and no gas-phase chemistry has occurred. The ISM SO $2 /$ OCS ratio is roughly an order of magnitude higher, which means that in the cold outer protostellar envelope there is more $\mathrm{SO}_{2}$ or less OCS in comparison to the warm protostellar phase of IRAS $16293-2422 \mathrm{~B}$. The $\mathrm{H}_{2} \mathrm{~S} / \mathrm{OCS}$ ratio is poorly constrained due to a lack of data on $\mathrm{H}_{2} \mathrm{~S}$ ice and gas. Based on the current limits, the ISM ratio can be lower (by an order of magnitude) or higher (by a factor of $\sim 1.3$ ) than that of source $B$. If the ISM $\mathrm{H}_{2} \mathrm{~S} / \mathrm{OCS}$ is indeed higher than that towards source $\mathrm{B}$, then there may indeed be less OCS in the cold outer protostellar envelope than in the warm protostellar phase.

\subsection{Comparison with 67P/C-G and ISM ices}

IRAS $16293-2422$ is thought to be analogous to our innate Solar Nebula, as it is one of the most chemically rich low-mass protostellar systems. Thanks to its proximity and the face-on orientation of the disc around source B, ALMA has been able to start precisely quantifying the chemistry of the disc-neighbourhood of B. Meanwhile, it has also been postulated that comets are the most pristine tracers of the innate cloud and protoplanetary disc that evolved into the Solar system that we have today. Therefore, it is interesting to compare the chemical inventories of comets to those of IRAS 16293-2422 B in order to quantify the chemical budgets of solar and extrasolar building blocks of planetary systems (Schöier et al.
2002; Bockelée-Morvan et al. 2015). The emission seen in the hot inner regions of the B protostellar core could potentially be tracing solid species that are just undergoing thermal desorption and thus, is in fact probing the hidden solid reservoir of planet-building material. The chemical composition of such building blocks will be shaped by many processes at different radii; however, this location likely uniquely probes the solid volatiles prior to modification in the gas phase.

Table 4 contains the molecular ratios relative to $\mathrm{H}_{2} \mathrm{~S}$ and OCS for IRAS $16293-2422 \mathrm{~B}$ in comparison to those of $67 \mathrm{P} / \mathrm{C}-\mathrm{G}$. The cometary values correspond to bulk coma quantities measured with DFMS/ROSINA and corrected for photodissociation and ionization by the radiation from the Sun (more important when in high proximity to the Sun and when the comet-spacecraft distance is large, e.g. $\sim 200 \mathrm{~km}$; table 3 of Calmonte et al. 2016), as obtained between equinox in May 2015 and perihelion in August 2015 (heliocentric distances between 1 and $2 \mathrm{au}$ ). The upper limits for $\mathrm{HS}_{2}$ and $\mathrm{H}_{2} \mathrm{~S}_{2}$ are based on October 2014 data, when $\mathrm{H}_{2} \mathrm{~S}$ abundance was maximized (sections 4.1.3 and 4.3.5 of Calmonte et al. 2016). The value given for $\mathrm{S}_{3}$ is an estimate based on the sole measurement obtained in March 2015 via a direct comparison of the measured ion current (largely uncertain, section 4.1.2 of Calmonte et al. 2016). The value tabulated for $\mathrm{H}_{2} \mathrm{CS}$ is based upon the sole clear signal at $46 u / e$ during a flyby in 2015 February, when the $\mathrm{CO}_{2}$ abundance was very low and the overlap with $\mathrm{C}^{16} \mathrm{O}^{18} \mathrm{O}$ could be avoided (private communication and section 4.2.3 of Calmonte et al. 2016). The value quoted for $\mathrm{CH}_{3} \mathrm{SH}$ is the mean of the four periods between the start of the mission and perihelion with a signal at $48 u / e$ corrected for photodissociation and ionization (section 4.3.4 of Calmonte et al. 2016). The value quoted for $\mathrm{C}_{2} \mathrm{H}_{5} \mathrm{SH}$ is the mean of four different periods between the start of the mission and perihelion with a signal at $62 u / e$ not corrected for photodissociation and ionization (sections 4.1.1 and 4.3.4 of Calmonte et al. 2016), which is highly uncertain due to the assumption of the entire $\mathrm{C}_{2} \mathrm{H}_{6} \mathrm{~S}$ peak being associated with ethyl mercaptan and the close overlap with the $\mathrm{C}_{5} \mathrm{H}_{2}$ fragment. The errors in Table 4 for the DFMS/ROSINA measurements stem from instrumental uncertainties (section 3.3 of Calmonte et al. 2016).

From the table, it can be seen that for OCS the ratio relative to $\mathrm{H}_{2} \mathrm{~S}$ for IRAS 16293-2422 B is a factor of 3-33 higher than that for 
Table 5. Molecular ratios as measured with these interferometric ALMA observations at the one beam offset position from source B of IRAS 16293-2422 and with the ROSINA instrument for 67P/C- $\mathrm{G}^{n}$.

\begin{tabular}{lcc}
\hline Ratio & ALMA B & $67 \mathrm{P} / \mathrm{C}-\mathrm{G}$ \\
\hline $\mathrm{SO} / \mathrm{SO}_{2}$ & 0.33 & $0.4-0.7$ (section 4.4 of Calmonte et al. 2016) \\
$\mathrm{CH}_{3} \mathrm{SH} / \mathrm{H}_{2} \mathrm{CS}$ & 3.7 & $0.69-5.2$ (based on values in Table 4) \\
$\mathrm{C}_{2} \mathrm{H}_{5} \mathrm{SH} / \mathrm{CH}_{3} \mathrm{SH}$ & $\leq 0.33^{p}$ & $0.0010-0.021$ (based on values in Table 4) \\
\hline
\end{tabular}

Notes. ${ }^{n}$ See Section 4.3 for the details on the ROSINA data.

${ }^{p}$ Using the average of the upper limits on the column densities of gauche- $\mathrm{C}_{2} \mathrm{H}_{5} \mathrm{SH}$ and anti- $\mathrm{C}_{2} \mathrm{H}_{5} \mathrm{SH}$.

$67 \mathrm{P} / \mathrm{C}-\mathrm{G}$, for $\mathrm{SO}$ a factor of $2-24$ lower, for $\mathrm{SO}_{2}$ a factor of $2-16$ lower and for $\mathrm{CH}_{3} \mathrm{SH}$ a factor of 1.2-12 lower. ${ }^{4}$ Relative to OCS, the IRAS $16293-2422 \mathrm{~B}$ ratios for $\mathrm{SO}$ and $\mathrm{SO}_{2}$ are $\sim 3$ orders of magnitude lower, and for $\mathrm{CH}_{3} \mathrm{SH}$ is $\sim 1$ order of magnitude lower. Such large differences are consistent with the fact that $\mathrm{H}_{2} \mathrm{~S}$ has not yet been detected in interstellar ices, but OCS has (Section 4.2).

These results indicate that in the case of IRAS 16293-2422 B there is significantly more OCS available, while for $67 \mathrm{P} / \mathrm{C}-\mathrm{G}$ more $\mathrm{H}_{2} \mathrm{~S}$ is present. Potentially, this has to do with the amount of solid carbon monoxide (CO) available in these systems. OCS may be more easily produced via grain-surface chemistry when $\mathrm{CO}$ ice is abundant, as seen in laboratory experiments of Ferrante et al. (2008). If so, then once $\mathrm{CO}$ undergoes thermal desorption into the gas-phase around $20 \mathrm{~K}$, the production of OCS would be inhibited (excluding any $\mathrm{CO}$ that maybe trapped in other ices). This could be an initial indication of our Solar system being born in a somewhat warmer, $>20 \mathrm{~K}$, environment rather than in the $10-15 \mathrm{~K}$ regime, which would lead to a deficiency of $\mathrm{CO}$ ice on the grains for the synthesis of OCS. This scenario is also favoured from the point of view of oxygen chemistry and the detection of $\mathrm{O}_{2}$ on $67 \mathrm{P} / \mathrm{C}-\mathrm{G}$ (Bieler et al. 2015; Taquet et al. 2016). In the case of IRAS 16293 $2422 \mathrm{~B}$, observations have shown its surrounding core to be very cold ( $\sim 12 \mathrm{~K}$; Menten et al. 1987), thus making CO ice plentiful in its birth cloud. It has been suggested that regions with elevated temperatures lead to $\mathrm{CO}$ ice-poor conditions, for example, the $\mathrm{R}$ $\mathrm{CrA}$ region with several low-mass protostars under the irradiation of an intermediate-mass protostar (Lindberg et al. 2014), and Orion hosting intermediate-mass protostars (Jørgensen et al. 2006).

A warmer environment would imply less efficient hydrogenation reactions, as the residence time of hydrogen atoms on the grains is reduced. This would make it difficult to produce $\mathrm{H}_{2} \mathrm{~S}$ through grainsurface atom-addition reactions. This molecule is predominantly formed via the associations of $\mathrm{H}$ and $\mathrm{S}$, followed by the reaction of HS with H (e.g. Furuya et al. 2015; Vidal et al. 2017). Some $\mathrm{H}_{2} \mathrm{~S}$ gas can also be produced via gas-phase channels at warmer temperatures. Temperature differences between birth clouds should also be noticeable in terms of complex organics. An initially warmer cloud would enhance mobility of heavy radicals on grain surfaces, such as $\mathrm{CH}_{3}$ and $\mathrm{SH}$, thereby boosting the abundance of complex species, such as $\mathrm{CH}_{3} \mathrm{SH}$ (although it is not exclusively formed via grain-surface chemistry). The $\mathrm{CH}_{3} \mathrm{SH} / \mathrm{H}_{2} \mathrm{CS}$ ratio for IRAS 16293 $2422 \mathrm{~B}$ is in range of the ratio for $67 \mathrm{P} / \mathrm{C}-\mathrm{G}$, given the errors in ROSINA data (differences by a factor of 0.7-5.3; Table 5). Hence, based the complex S-bearing species $\mathrm{CH}_{3} \mathrm{SH}$ relative to $\mathrm{H}_{2} \mathrm{CS}$, it is not possible to conclusively say which target is richer in complex

\footnotetext{
${ }^{4}$ This contradicts section 5.2 of Calmonte et al. (2016) due to different assumptions on the quantity of $\mathrm{H}_{2} \mathrm{~S}$ and the use of abundances versus column densities.
}

organic molecules. Given the large differences in the quantities of $\mathrm{H}_{2} \mathrm{~S}$ and OCS between the two data sets, ratios relative to them are less meaningful. As it has only been possible to derive an upper limit for $\mathrm{C}_{2} \mathrm{H}_{5} \mathrm{SH}$, the $\mathrm{C}_{2} \mathrm{H}_{5} \mathrm{SH} / \mathrm{CH}_{3} \mathrm{SH}$ ratio cannot yet be used to compare the budgets of S-bearing complex species between IRAS 16293-2422 B and 67P/C-G. A rigorous comparison between IRAS 16293-2422 B and 67P/C-G in terms of a larger set of other $\mathrm{O}-$ and $\mathrm{N}$-bearing complex organics is the subject of a dedicated paper.

Additional physics could be at play here - the degree of irradiation that the two targets are subjected to. Our Sun is an isolated star that is thought to have formed in a medium-sized stellar cluster of $10^{3}-10^{4}$ members (Adams 2010). Laboratory experiments of Chen et al. (2015) have shown rapid conversion of $\mathrm{H}_{2} \mathrm{~S}$ into OCS upon VUV and EUV irradiation. If IRAS 16293-2422 B experienced higher UV fluxes, perhaps due to its binary A companion, then that may have stimulated the conversion of its $\mathrm{H}_{2} \mathrm{~S}$ reservoir into OCS. In addition, higher levels of UV would explain the higher fraction of complex S-bearing species relative to $\mathrm{H}_{2} \mathrm{CS}$ of IRAS 16293-2422 $\mathrm{B}$, since UV enhances the availability of radicals in the ice and boosts grain-surface chemistry. This scenario would also correlate with OCS forming during the collapse phases and there, thus, being less OCS in ISM ices (Section 4.2; although the presence of $\mathrm{H}_{2} \mathrm{~S}$ ice is yet to be demonstrated and quantified in prestellar sources).

Another diagnostic independent of the $\mathrm{H}_{2} \mathrm{~S}$ and OCS quantities is the $\mathrm{SO} / \mathrm{SO}_{2}$ ratio. Based on the presented PILS Band 7 data, it is 0.33 for IRAS 16293-2422 B; and based on the ROSINA data, it is in the 0.4-0.7 range for 67P/C-G (Table 5). Such excellent (a factor of $\sim 2$ ) agreement could point to both data sets sampling comparable materials. If the quantities of $\mathrm{SO}$ and $\mathrm{SO}_{2}$ are set before and/or during the prestellar stage, as supported by the detection of $\mathrm{SO}_{2}$ as an ISM ice (Section 4.2), then this again points to IRAS 16293 2422 B and 67P/C-G having formed from similar birth clouds. Unfortunately, even with JWST it may be impossible to quantify the ISM SO/SO 2 ice ratio due to the overlap of their symmetric stretching modes. Gas-phase routes to $\mathrm{SO}$ and $\mathrm{SO}_{2}$ via reactions of $\mathrm{OH}$ and $\mathrm{O}_{2}$ with $\mathrm{S}$ at warm temperatures may also skew the ratio.

The ROSINA instrument determined that $\sim 27$ per cent of the sulphur is in the atomic form and constitutes the second largest sulphur reservoir after $\mathrm{H}_{2} \mathrm{~S}$ (fig. 16 of Calmonte et al. 2016). It was also argued that five times more $\mathrm{H}_{2} \mathrm{~S}$ than detected would be necessary to account for the measured quantity of atomic sulphur; hence, it is indeed stored in its atomic form in 67P/C-G (Calmonte et al. 2016). Unfortunately, atomic sulphur does not have any lines in the frequency range covered by the PILS Band 7 data. 67P/C-G has been observed to contain sulphur-chain molecules like $S_{2}, S_{3}$, and $S_{4}$, which could, in part, be fragments of even heavier $S_{n}$ species (up to $n=8$ ). The upper limits derived in this work are less constraining than the quantities measured for $67 \mathrm{P} / \mathrm{C}-\mathrm{G}$ (Table 4). ROSINA did not (conclusively) detect $\mathrm{HS}_{2}$ and $\mathrm{H}_{2} \mathrm{~S}_{2}$ (section 4.1.3 


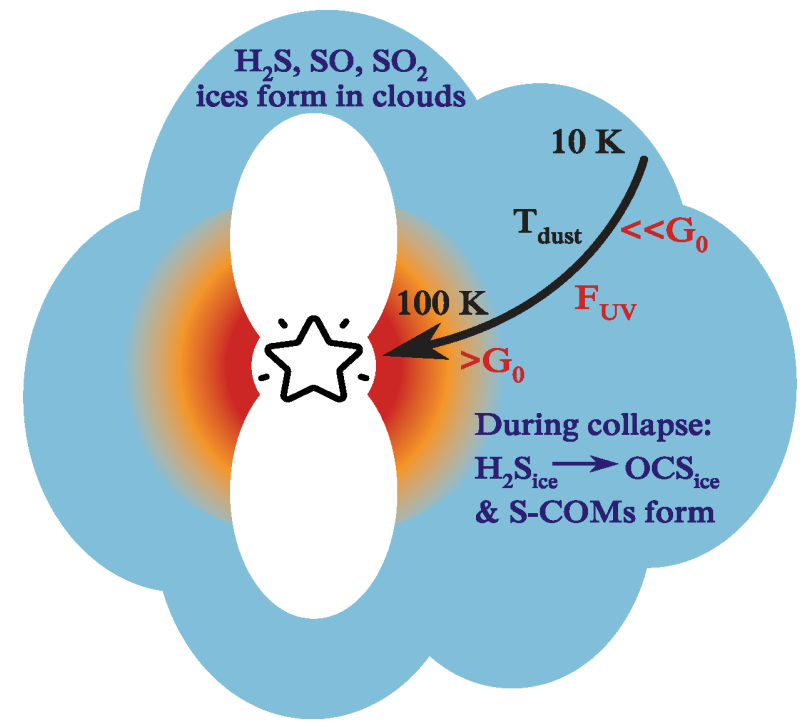

Figure 2. A schematic summarizing the chemical evolution of S-bearing species during the earliest phases of low-mass star formation. S-COMs stand for S-bearing complex organic molecules. $\mathrm{G}_{0}$ is the interstellar FUV radiation field of $1.6 \times 10^{-3} \mathrm{erg} \mathrm{cm}^{-2} \mathrm{~s}^{-1}$ (Habing 1968).

of Calmonte et al. 2016). These species were not detected towards IRAS 16293-2422 B with the PILS Band 7 data either. It was not possible to derive the abundance of CS with ROSINA, due to interference with $\mathrm{CO}_{2}$; and due to it being a common fragment of other sulphur-bearing species. Hence, it cannot be compared in a meaningful fashion with the data in this work. Finally, as $\mathrm{CS}_{2}$ is a linear symmetric molecule without strong rotational lines, it is not possible to search for it in the PILS Band 7 data; even though it has been clearly detected with mass spectrometry on $67 \mathrm{P} / \mathrm{C}-\mathrm{G}$.

The findings of this work can be summarized in Fig. 2. It seems that the abundances of $\mathrm{SO}, \mathrm{SO}_{2}$, and $\mathrm{H}_{2} \mathrm{~S}$ are set in the translucent cloud and prestellar core phases, prior to the onset of collapse. This is supported by the similar relative ratios between the PILS data on IRAS 16293-2422 B, the ISM ices towards W33A (Section 4.2) and the ROSINA data on $67 \mathrm{P} / \mathrm{C}-\mathrm{G}$. The differences between the data sets can be explained in terms of the amount of UV radiation that the materials are exposed to, which occurs during the collapse of the system, already in the earliest embedded stages, as well as the temperature of the birth cloud. Irradiation brings about the conversion of $\mathrm{H}_{2} \mathrm{~S}$ into OCS and the formation of S-bearing complex organic molecules. This explains the richer chemistry towards IRAS 16293-2422 B and our Solar system, in comparison to cold outer protostellar envelopes. Whether IRAS 16293-2422 B is relatively chemically richer than our Solar system as a result of higher UV fluxes based on its binary nature still remains to be conclusively tested via a larger set of complex organic species. If this is shown to be the case, then this would correlate with IRAS 16293-2422 being one of the most chemically rich low-mass protostars; and imply that our Solar system is not special chemically speaking.

\section{CONCLUSIONS}

In this paper, the sulphur inventory at the one beam offset position from source B in the SW direction in the binary protostellar system IRAS 16293-2422 has been presented. The ALMA Band 7 data analysed are part of the PILS survey towards this target. Sulphur is thought to be a unique simultaneous tracer of both the volatile and refractory components. By comparing molecular ratios observed towards IRAS 16293-2422 B - a Solar system proxy, and those obtained for $67 \mathrm{P} / \mathrm{C}-\mathrm{G}$ - a pristine tracer of the innate Solar Nebula, the chemical links between the early embedded protostellar phases and the protoplanetary building blocks can be explored. The main conclusions of this paper are as follows:

(i) The sulphur-bearing species previously detected towards IRAS 16293-2422 B have now been firmly detected towards the one beam offset position and spatially resolved with ALMA: $\mathrm{SO}_{2}$ in the $v=0,{ }^{34} \mathrm{SO}_{2}$, OCS in the $v_{2}=1$ state, $\mathrm{O}^{13} \mathrm{CS}, \mathrm{OC}^{34} \mathrm{~S}, \mathrm{OC}^{33} \mathrm{~S}$ (first-time detection towards this source), ${ }^{18} \mathrm{OCS}, \mathrm{H}_{2} \mathrm{CS}$, HDCS, and $\mathrm{CH}_{3} \mathrm{SH}$ in the $v=0-2$ state. Furthermore, several are detected tentatively due to a lack of lines and/or blending: SO in the $v=0$ state, OCS in the $v=0$ state, $\mathrm{C}^{34} \mathrm{~S}$ in the $v=0,1$ state, $\mathrm{C}^{33} \mathrm{~S}$ in the $v=0,1$ state, $\mathrm{C}^{36} \mathrm{~S}$ (tentative first time detection towards a low-mass protostar), HDS and $\mathrm{HD}^{34} \mathrm{~S}$. All the lines from these sulphur-bearing molecules are narrow $\left(\sim 1 \mathrm{~km} \mathrm{~s}^{-1}\right)$ and are probing the small disc scales of source B. Unfortunately, the unknown $\mathrm{HDS} / \mathrm{H}_{2} \mathrm{~S}$ ratio leaves the column density of $\mathrm{H}_{2} \mathrm{~S}$ uncertain by at least an order of magnitude.

(ii) In comparison to earlier single dish observations, the molecular ratios determined from interferometric data can be up to four orders of magnitude lower. On large scales, $\mathrm{SO}$ and $\mathrm{SO}_{2}$ likely emit from outflows; and OCS, $\mathrm{H}_{2} \mathrm{~S}$, and $\mathrm{H}_{2} \mathrm{CS}$ originate from the envelope. All these S-bearing species are also present on the small discand inner envelope-scales. Single dish data may be dominated by source A, which is bright in emission from S-bearing molecules.

(iii) In comparison to ROSINA measurements of the bulk volatile composition of 67P/C-G, the molecular ratios of IRAS 16293-2422 $\mathrm{B}$ can differ significantly, potentially by several orders of magnitude. In particular, IRAS 16293-2422 B contains much more OCS than $\mathrm{H}_{2} \mathrm{~S}$. However, the $\mathrm{SO} / \mathrm{SO}_{2}$ is in close agreement between the two targets.

(iv) The agreement in terms of $\mathrm{SO}, \mathrm{SO}_{2}$, the disagreement in terms of $\mathrm{H}_{2} \mathrm{~S}$ and OCS, and comparable (differences by a factor of 0.7-5.3) $\mathrm{CH}_{3} \mathrm{SH} / \mathrm{H}_{2} \mathrm{CS}$ ratios towards IRAS $16293-2422 \mathrm{~B}$ in comparison to that of our Solar system (as probed by $67 \mathrm{P} / \mathrm{C}-\mathrm{G}$ ) may stem from different levels of UV irradiation during the initial collapse of the systems. Potentially higher UV levels near source B, as a result of its binary structure, may lead to the conversion of $\mathrm{H}_{2} \mathrm{~S}$ into OCS and enhance the formation of S-bearing complex organic molecules. An initially warmer birth cloud may also contribute to the lower quantities of OCS in the Solar system by reducing the amount of available $\mathrm{CO}$ ice.

The results also highlight the importance of the level of UV exposure and temperature of the parental clouds in determining the physical and chemical structures of low-mass protostars. Given that these may vary significantly from region to region (see e.g. review by Adams 2010), it is also likely that significant source-tosource variations in chemistry may be found. Future studies will also explore the protostellar-cometary connection via isotopic ratios and a full set of complex organic species, thereby isolating chemical links formed during cold phases of evolution that are dominated by grain-surface chemistry from those occurring during warmer, irradiated phases of collapse.

\section{ACKNOWLEDGEMENTS}

The authors would like to thank Dr. Catherine Walsh, Dr. Vianney Taquet, Mr. Ko-Ju Chuang, and Dr. Martin Rubin for useful discussions on sulphur chemistry and ROSINA measurements. This work 
is supported by a Huygens fellowship from Leiden University, the European Union A-ERC grant 291141 CHEMPLAN, the Netherlands Research School for Astronomy (NOVA), a Royal Netherlands Academy of Arts and Sciences (KNAW) professor prize, the Center for Space and Habitability (CSH) Fellowship, and the IAU Gruber Foundation Fellowship.

The research of $\mathrm{JKJ}$ and his group is supported by the European Research Council (ERC) under the European Union's Horizon 2020 research and innovation programme (grant agreement No 646908) through ERC Consolidator Grant 'S4F'. Research at the Centre for Star and Planet Formation is funded by the Danish National Research Foundation. A.C. postdoctoral grant is funded by the ERC Starting Grant 3DICE (grant agreement 336474).

This paper makes use of the following ALMA data: ADS/JAO.ALMA\#2013.1.00278.S. ALMA is a partnership of ESO (representing its member states), NSF (USA) and NINS (Japan), together with NRC (Canada), MOST and ASIAA (Taiwan), and KASI (Republic of Korea), in cooperation with the Republic of Chile. The Joint ALMA Observatory is operated by ESO, AUI/NRAO and NAOJ.

\section{REFERENCES}

A'Hearn M. F., Schleicher D. G., Feldman P. D., 1983, ApJ, 274, L99

Adams F. C., 2010, ARA\&A, 48, 47

Ahrens V., Winnewisser G., 1999, Z. Nat.forsch. A, 54, 131

Aikawa Y. et al., 2012, A\&A, 538, A57

Altwegg K. et al., 2017, MNRAS, 469, S130

Anderson D. E., Bergin E. A., Maret S., Wakelam V., 2013, ApJ, 779, 141

Asplund M., Grevesse N., Sauval A. J., Scott P., 2009, ARA\&A, 47, 481

Balsiger H. et al., 2007, Space Sci. Rev., 128, 745

Baryshev A. M. et al., 2015, A\&A, 577, A129

Belloche A., Müller H. S. P., Menten K. M., Schilke P., Comito C., 2013, A\&A, 559, A47

Belov S. P., Yamada K. M. T., Winnewisser G., Poteau L., Bocquet R., Demaison J., Polyansky O., Tretyakov M. Y., 1995, J. Mol. Spectrosc., 173,380

Belov S. P., Tretyakov M. Y., Kozin I. N., Klisch E., Winnewisser G., Lafferty W. J., Flaud J.-M., 1998, J. Mol. Spectrosc., 191, 17

Bettens F. L., Sastry K. V. L. N., Herbst E., Albert S., Oesterling L. C., De Lucia F. C., 1999, ApJ, 510, 789

Bieler A. et al., 2015, Nature, 526, 678

Biver N. et al., 2016, A\&A, 589, A78

Blake G. A., van Dishoeck E. F., Sargent A. I., 1992, ApJ, 391, L99

Blake G. A., van Dishoeck E. F., Jansen D. J., Groesbeck T. D., Mundy L. G., 1994, ApJ, 428, 680

Bockelée-Morvan D. et al., 2015, A\&A, 583, A6

Bogey M., Demuynck C., Destombes J. L., 1982, J. Mol. Spectrosc., 95, 35

Bogey M., Civiš S., Delcroix B., Demuynck C., Krupnov A. F., Quiguer J., Tretyakov M. Y., Walters A., 1997, J. Mol. Spectrosc., 182, 85

Boogert A. C. A., Schutte W. A., Helmich F. P., Tielens A. G. G. M., Wooden D. H., 1997, A\&A, 317, 929

Boogert A. C. A., Gerakines P. A., Whittet D. C. B., 2015, ARA\&A, 53, 541

Calmonte U. et al., 2016, MNRAS, 462, S253

Calmonte U. et al., 2017, MNRAS, 469, S787

Camy-Peyret C., Flaud J.-M., Lechuga-Fossat L., Johns J. W. C., 1985, J. Mol. Spectrosc., 109, 300

Caux E. et al., 2011, A\&A, 532, A23

Cernicharo J., Marcelino N., Roueff E., Gerin M., Jiménez-Escobar A., Muñoz Caro G. M., 2012, ApJ, 759, L43

Chen Y.-J. et al., 2015, ApJ, 798, 80

Coutens A. et al., 2016, A\&A, 590, L6

Drdla K., Knapp G. R., van Dishoeck E. F., 1989, ApJ, 345, 815
Dubrulle A., Demaison J., Burie J., Boucher D., 1980, Z. Nat.forsch. A, 35, 471

Duley W. W., Millar T. J., Williams D. A., 1980, MNRAS, 192, 945

Esplugues G. B., Viti S., Goicoechea J. R., Cernicharo J., 2014, A\&A, 567, A95

Fabricant B., Krieger D., Muenter J. S., 1977, J. Chem. Phys., 67, 1576

Ferrante R. F., Moore M. H., Spiliotis M. M., Hudson R. L., 2008, ApJ, 684, 1210

Furuya K., Aikawa Y., Hincelin U., Hassel G. E., Bergin E. A., Vasyunin A. I., Herbst E., 2015, A\&A, 584, A124

Geiss J., 1988, in Klare G., ed., Reviews in Modern Astronomy, Vol. 1, Reviews in Modern Astronomy. p. 1

Gibb E. L. et al., 2000, ApJ, 536, 347

Glassmeier K.-H., Boehnhardt H., Koschny D., Kührt E., Richter I., 2007, Space Sci. Rev., 128, 1

Goesmann F. et al., 2015, Science, 349, 020689

Goicoechea J. R., Pety J., Gerin M., Teyssier D., Roueff E., Hily-Blant P., Baek S., 2006, A\&A, 456, 565

Golubiatnikov G. Y., Lapinov A. V., Guarnieri A., Knöchel R., 2005, J. Mol. Spectrosc., 234, 190

Grim R. J. A., Greenberg J. M., 1987, A\&A, 181, 155

Habing H. J., 1968, Bull. Astr. Inst. Neth., 19, 421

Hasegawa T. et al., 1984, ApJ, 283, 117

Helminger P., Cook R. L., De Lucia F. C., 1971, J. Mol. Spectrosc., 40, 125

Hillger R. E., Strandberg M. W., 1951, Phys. Rev., 83, 575

Hirahara Y. et al., 1992, ApJ, 394, 539

Jansen D. J., van Dishoeck E. F., Black J. H., 1994, A\&A, 282, 605

Jefferts K. B., Penzias A. A., Wilson R. W., Solomon P. M., 1971, ApJ, 168 , L111

Jessberger E. K., Christoforidis A., Kissel J., 1988, Nature, 332, 691

Jessup K. L., Spencer J., Yelle R., 2007, Icarus, 192, 24

Jørgensen J. K., Johnstone D., van Dishoeck E. F., Doty S. D., 2006, A\&A, 449,609

Jørgensen J. K., Bourke T. L., Nguyen Luong Q., Takakuwa S., 2011, A\&A, 534, A100

Jørgensen J. K., Favre C., Bisschop S. E., Bourke T. L., van Dishoeck E. F., Schmalzl M., 2012, ApJ, 757, L4

Jørgensen J. K. et al., 2016, A\&A, 595, A117

Jørgensen J. K. et al., 2017, A\&A, submitted

Kolesniková L., Tercero B., Cernicharo J., Alonso J. L., Daly A. M., Gordon B. P., Shipman S. T., 2014, ApJ, 784, L7

Le Roy L. et al., 2015, A\&A, 583, A1

Leen T. M., Graff M. M., 1988, ApJ, 325, 411

Ligterink N. F. W. et al., 2017, MNRAS, 469, 2219

Lindberg J. E., Jørgensen J. K., Green J. D., Herczeg G. J., Dionatos O., Evans N. J., Karska A., Wampfler S. F., 2014, A\&A, 565, A29

Linke R. A., Frerking M. A., Thaddeus P., 1979, ApJ, 234, L139

Loinard L., Torres R. M., Mioduszewski A. J., Rodríguez L. F., 2008, ApJ, 675, L29

Lucas R., Liszt H. S., 2002, A\&A, 384, 1054

Lykke J. M. et al., 2017, A\&A, 597, A53

Maeda A. et al., 2008, ApJS, 176, 543

Majumdar L., Gratier P., Vidal T., Wakelam V., Loison J.-C., Hickson K. M., Caux E., 2016, MNRAS, 458, 1859

Martín-Doménech R., Jiménez-Serra I., Muñoz Caro G. M., Müller H. S. P., Occhiogrosso A., Testi L., Woods P. M., Viti S., 2016, A\&A, 585, A112

Mauersberger R., Henkel C., Langer N., Chin Y.-N., 1996, A\&A, 313, L1

Menten K. M., Serabyn E., Guesten R., Wilson T. L., 1987, A\&A, 177, L57

Millar T. J., Herbst E., 1990, A\&A, 231, 466

Minh Y. C., Irvine W. M., McGonagle D., Ziurys L. M., 1990, ApJ, 360, 136

Minowa H., Satake M., Hirota T., Yamamoto S., Ohishi M., Kaifu N., 1997, ApJ, 491, L63

Mitchell G. F., 1984, ApJ, 287, 665

Morino I., Yamada K. M. T., Maki A. G., 2000, J. Mol. Spectrosc., 200, 145

Moullet A., Lellouch E., Moreno R., Gurwell M. A., Moore C., 2008, A\&A, 482,279 
Moullet A., Lellouch E., Moreno R., Gurwell M., Black J. H., Butler B., 2013, ApJ, 776, 32

Müller H. S. P., Brünken S., 2005, J. Mol. Spectrosc., 232, 213

Müller H. S. P., Schlöder F., Stutzki J., Winnewisser G., 2005, J. Mol. Spectrosc., 742, 215

Müller H. S. P. et al., 2016, A\&A, 587, A92

Mumma M. J., Charnley S. B., 2011, ARA\&A, 49, 471

Murillo N. M., van Dishoeck E. F., van der Wiel M. H. D., Jørgensen J. K., Drozdovskaya M. N., Calcutt H., Harsono D., 2017, A\&A, submitted Oppenheimer M., Dalgarno A., 1974, ApJ, 187, 231

Ortiz-León G. N. et al., 2017, ApJ, 834, 141

Oya Y., Sakai N., López-Sepulcre A., Watanabe Y., Ceccarelli C., Lefloch B., Favre C., Yamamoto S., 2016, ApJ, 824, 88

Palumbo M. E., Tielens A. G. G. M., Tokunaga A. T., 1995, ApJ, 449, 674

Palumbo M. E., Geballe T. R., Tielens A. G. G. M., 1997, ApJ, 479, 839

Pastor J., Buj J., Estalella R., Lopez R., Anglada G., Planesas P., 1991, A\&A, 252,320

Patel D., Margolese D., Dyke T. R., 1979, J. Chem. Phys., 70, 2740

Penzias A. A., Solomon P. M., Wilson R. W., Jefferts K. B., 1971, ApJ, 168, L53

Persson M. V. et al., 2018, A\&A, 610, A54

Pineau des Forets G., Roueff E., Flower D. R., 1986, MNRAS, 223, 743

Pineda J. E. et al., 2012, A\&A, 544, L7

Powell F. X., Lide D. R., Jr, 1964, J. Chem. Phys., 41, 1413

Prasad S. S., Huntress W. T., Jr, 1982, ApJ, 260, 590

Rotundi A. et al., 2015, Science, 347, aaa3905

Ruffle D. P., Hartquist T. W., Caselli P., Williams D. A., 1999, MNRAS, 306, 691

Sakai N. et al., 2014, Nature, 507, 78

Schöier F. L., Jørgensen J. K., van Dishoeck E. F., Blake G. A., 2002, A\&A, 390, 1001

Smith D., Adams N. G., Giles K., Herbst E., 1988, A\&A, 200, 191

Tanaka K., Tanaka T., Suzuki I., 1985, J. Chem. Phys., 82, 2835

Taquet V., Furuya K., Walsh C., van Dishoeck E. F., 2016, MNRAS, 462, S99

Tsunekawa S., Taniguchi I., Tambo A., Nagai T., Kojima T., Nakagawa K., 1989, J. Mol. Spectrosc., 134, 63

Turner B. E., 1992, ApJ, 396, L107

van der Tak F. F. S., Boonman A. M. S., Braakman R., van Dishoeck E. F., 2003, A\&A, 412, 133

van Dishoeck E. F., Blake G. A., Jansen D. J., Groesbeck T. D., 1995, ApJ, 447,760

Vidal T. H. G., Loison J.-C., Jaziri A. Y., Ruaud M., Gratier P., Wakelam V., 2017, MNRAS, 469, 435

Viswanathan R., Dyke T. R., 1984, J. Mol. Spectrosc., 103, 231

Watt G. D., Charnley S. B., 1985, MNRAS, 213, 157

Wilson T. L., 1999, Rep. Prog. Phys., 62, 143

Wilson T. L., Rood R., 1994, ARA\&A, 32, 191

Winnewisser G., Cook R. L., 1968, J. Mol. Spectrosc., 28, 266

Wlodek S., Bohme D. K., Herbst E., 1988, MNRAS, 235, 493

Woods P. M., Occhiogrosso A., Viti S., Kaňuchová Z., Palumbo M. E., Price S. D., 2015, MNRAS, 450, 1256

Xu L.-H. et al., 2012, J. Chem. Phys., 137, 104313

Zapata L. A., Palau A., Galván-Madrid R., Rodríguez L. F., Garay G., Moran J. M., Franco-Hernández R., 2015, MNRAS, 447, 1826

\section{SUPPORTING INFORMATION}

Supplementary data are available at MNRAS online.

\section{Figures B1-B41.}

Please note: Oxford University Press is not responsible for the content or functionality of any supporting materials supplied by the authors. Any queries (other than missing material) should be directed to the corresponding author for the article.

\section{APPENDIX A: PARTITION OF SULPHUR ON 67P/C-G}

For the moment, the data on elemental abundances in the dust of $67 \mathrm{P} / \mathrm{C}-\mathrm{G}$ has not been released by the Cometary Secondary Ion Mass Spectrometer (COSIMA) team. Therefore, the partition of sulphur between the refractory (dust) and volatile (ice) components has been derived based upon the information on the $S / O$ ratio in volatiles as derived by the ROSINA team and the results obtained for comet 1P/Halley (Jessberger, Christoforidis \& Kissel 1988).

For comet 1P/Halley, it has been shown that the dust-to-ice mass ratio is

$m_{\text {dust } 1 \mathrm{P}} / m_{\text {icelP }}=2$,

so then

$m_{\text {comet } 1 P}=m_{\text {dust } 1 \mathrm{P}}+m_{\text {icelP }}=3 m_{\text {icelP }}=\frac{3}{2} m_{\text {dust } 1 \mathrm{P}}$.

In other terms,

$m_{\text {dust } 1 \mathrm{P}}=\frac{2}{3} m_{\text {comet } 1 \mathrm{P}}$

and

$m_{\text {ice } 1 \mathrm{P}}=\frac{1}{3} m_{\text {comet } 1 \mathrm{P}}$.

According to Geiss (1988), 23 per cent of all the oxygen atoms on $1 \mathrm{P} /$ Halley is in the refractory (dust) component by number, i.e.

$n_{\mathrm{O}_{\text {dust } 1 \mathrm{P}}}=0.23 n_{\mathrm{O}_{\text {tot } 1 \mathrm{P}}}$,

where $n$ is the total number of atoms and is dimensionless. So,

$n_{\mathrm{O}_{\text {icelP }}}=0.77 n_{\mathrm{O}_{\text {tot } 1 \mathrm{P}}}$.

Then the number densities (in $\mathrm{cm}^{-3}$ ) of oxygen in the refractory (dust) and volatile (ice) components of 1P/Halley are given by

$\rho_{\mathrm{O}_{\text {dust } 1 \mathrm{P}}}=\frac{n_{\mathrm{O}_{\text {dust } 1 \mathrm{P}}}}{m_{\mathrm{O}_{\text {dust } 1 \mathrm{P}}}} \times P_{\mathrm{O}_{\text {dust } 1 \mathrm{P}}}=\frac{0.23 n_{\mathrm{O}_{\text {tot } 1 \mathrm{P}}}}{\frac{2}{3} m_{\mathrm{O}_{\text {comet IP }}}} \times P_{\mathrm{O}_{\text {dust } 1 \mathrm{P}}}$,

$\rho_{\mathrm{O}_{\text {icelP }}}=\frac{n_{\mathrm{O}_{\text {icelP }}}}{m_{\mathrm{O}_{\text {icelP }}}} \times P_{\mathrm{O}_{\text {icelP }}}=\frac{0.77 n_{\mathrm{O}_{\text {totlP }}}}{\frac{1}{3} m_{\mathrm{O}_{\text {comet IP }}}} \times P_{\mathrm{O}_{\text {icelP }}}$,

where $\rho$ is the mass density (in $\mathrm{g} \mathrm{cm}^{-3}$ ). The ratio of the two is

$\rho_{\mathrm{O}_{\text {icelP }}} / \rho_{\mathrm{O}_{\text {dust IP }}}=\frac{0.77 \times 2}{0.23} \times \frac{P_{\mathrm{O}_{\text {icelP }}}}{P_{\mathrm{O}_{\text {dust } 1 \mathrm{P}}}} \approx 6.7 \frac{P_{\mathrm{O}_{\text {icelP }}}}{P_{\mathrm{O}_{\text {dust } 1 \mathrm{P}}}}$.

Now let us assume that $1 \mathrm{P} / \mathrm{Halley}$ and $67 \mathrm{P} / \mathrm{C}-\mathrm{G}$ have been made from the same dust and ice. This means that $\rho_{\mathrm{O}_{\text {icelP }}}=\rho_{\mathrm{O}_{\text {ice67P }}}$, $\rho_{\mathrm{O}_{\text {dust } 1 \mathrm{P}}}=\rho_{\mathrm{O}_{\text {dust67P }}}$ and that $P_{\mathrm{O}_{\text {icelP }}}=P_{\mathrm{O}_{\text {ice67P }}}, P_{\mathrm{O}_{\text {dust } 1 \mathrm{P}}}=P_{\mathrm{O}_{\text {dust67P }}}$. Then it also follows that

$\rho_{\mathrm{O}_{\text {ice67P }}} / \rho_{\mathrm{O}_{\text {dust } 67 \mathrm{P}}} \approx 6.7 \frac{P_{\mathrm{O}_{\text {ice67P }}}}{P_{\mathrm{O}_{\text {dust } 67 \mathrm{P}}}}$.

For comet 67P/C-G, Rotundi et al. (2015) derived that the dust-toice mass ratio is

$m_{\text {dust67P }} / m_{\text {ice67P }}=4$,

so then

$m_{\text {comet67P }}=m_{\text {dust67P }}+m_{\text {ice67P }}=5 m_{\text {ice67P }}=\frac{5}{4} m_{\text {dust67P }}$.

In other terms,

$m_{\text {dust67P }}=\frac{4}{5} m_{\text {comet67P }}$ 
and

$m_{\text {ice67P }}=\frac{1}{5} m_{\text {comet67P. }}$

Subsequently, the number of oxygen atoms in the refractory (dust) and volatile (ice) components of $67 \mathrm{P} / \mathrm{C}-\mathrm{G}$ become

$$
\begin{aligned}
n_{\mathrm{O}_{\text {dust } 67 \mathrm{P}}} & =\rho_{\mathrm{O}_{\text {dust } 67 \mathrm{P}}} \times \frac{m_{\mathrm{O}_{\text {dust } 67 \mathrm{P}}}}{P_{\mathrm{O}_{\text {dust } 67 \mathrm{P}}}} \\
& =\rho_{\mathrm{O}_{\text {dust } 67 \mathrm{P}}} \times \frac{\frac{4}{5} m_{\text {comet67P }}}{P_{\mathrm{O}_{\text {dust } 67 \mathrm{P}}}}, \\
n_{\mathrm{O}_{\text {ice67P }}} & =\rho_{\mathrm{O}_{\text {ice67P }}} \times \frac{m_{\mathrm{O}_{\text {ice67P }}}}{P_{\mathrm{O}_{\text {ice67P }}}} \\
& =\rho_{\mathrm{O}_{\text {ice67P }}} \times \frac{\frac{1}{5} m_{\text {comet67P }}}{P_{\mathrm{O}_{\text {ice67P }}}},
\end{aligned}
$$

and the ratio of the two is

$$
\begin{aligned}
n_{\mathrm{O}_{\text {dust } 67 \mathrm{P}}} / n_{\mathrm{O}_{\text {ice67P }}} & =4 \times \frac{\rho_{\mathrm{O}_{\text {dust67P }}}}{\rho_{\mathrm{O}_{\text {ice67P }}}} \times \frac{P_{\mathrm{O}_{\text {ice67P }}}}{P_{\mathrm{O}_{\text {dust67P }}}} \\
& \approx 4 \times \frac{P_{\mathrm{O}_{\text {dust67P }}}}{6.7 \times P_{\mathrm{O}_{\text {ice67P }}}} \times \frac{P_{\mathrm{O}_{\text {ice67P }}}}{P_{\mathrm{O}_{\text {dust67P }}}} \approx 0.60 .
\end{aligned}
$$

Hence,

$$
n_{\mathrm{O}_{\text {comet67P }}}=n_{\mathrm{O}_{\text {dust } 67 \mathrm{P}}}+n_{\mathrm{O}_{\text {ice67P }}}=1.60 n_{\mathrm{O}_{\text {ice67P }}}=2.7 n_{\mathrm{O}_{\text {dust } 67 \mathrm{P}}} \text {. }
$$

This implies that 37 per cent of all the oxygen atoms on $67 \mathrm{P} / \mathrm{C}-\mathrm{G}$ is in the refractory (dust) component by number; and that 63 per cent of all the oxygen atoms on $67 \mathrm{P} / \mathrm{C}-\mathrm{G}$ is in the volatile (ice) component by number.

It has also been shown by Jessberger et al. (1988) that S/O in the dust of $1 \mathrm{P} / \mathrm{Halley}$ is 8.1 per cent (based on values in table 1). Assuming again that $1 \mathrm{P} /$ Halley and $67 \mathrm{P} / \mathrm{C}-\mathrm{G}$ have been made from the same dust, i.e.

$$
(\mathrm{S} / \mathrm{O})_{\mathrm{dust} 67 \mathrm{P}}=(\mathrm{S} / \mathrm{O})_{\mathrm{dust} 1 \mathrm{P}}=8.1 \text { per cent; }
$$

then the overall $\mathrm{S} / \mathrm{O}$ ratio in the dust and ice of $67 \mathrm{P} / \mathrm{C}-\mathrm{G}$ is

$$
\begin{aligned}
(\mathrm{S} / \mathrm{O})_{\text {comet67P }} & =\frac{n_{\mathrm{O}_{\text {dust } 67 \mathrm{P}}}}{n_{\mathrm{O}_{\text {comet67P }}}}(\mathrm{S} / \mathrm{O})_{\text {dust67P }}+\frac{n_{\mathrm{O}_{\text {ice67P }}}}{n_{\mathrm{O}_{\text {comet } 67 \mathrm{P}}}}(\mathrm{S} / \mathrm{O})_{\text {ice67P }} \\
& =0.37 \times 8.1 \text { per cent }+0.63 \times 1.47 \text { per cent } \\
& \approx 3.9 \text { per cent }
\end{aligned}
$$

where the $\mathrm{S} / \mathrm{O}$ in the ice of $67 \mathrm{P} / \mathrm{C}-\mathrm{G}$ of 1.47 per cent has been used (as given in section 5.6 of Calmonte et al. 2016) as derived from ROSINA data.

If $(\mathrm{S} / \mathrm{O})_{\text {comet67P }}=3.9$ per cent and $(\mathrm{S} / \mathrm{O})_{\text {ice67P }}=1.47$ percent, then

$$
\begin{aligned}
& \frac{(\mathrm{S} / \mathrm{O})_{\text {ice67P }}}{(\mathrm{S} / \mathrm{O})_{\text {comet67P }}}=\frac{1.47}{3.9}, \\
& \frac{n_{\mathrm{S}_{\text {ice67P }}}}{n_{\mathrm{S}_{\text {comet67P }}}} \times \frac{n_{\mathrm{O}_{\text {comet67P }}}}{n_{\mathrm{O}_{\text {ice67P }}}}=\frac{1.47}{3.9}, \\
& \frac{n_{\mathrm{S}_{\text {ice67P }}}}{n_{\mathrm{S}_{\text {comet67P }}}} \times 1.6=\frac{1.47}{3.9}, \\
& \frac{n_{\mathrm{S}_{\text {ice67P }}}}{n_{\mathrm{S}_{\text {comet67P }}}}=\frac{1.47}{3.9 \times 1.6}=0.24 .
\end{aligned}
$$

This means that 24 per cent of all the sulphur atoms on $67 \mathrm{P} / \mathrm{C}-\mathrm{G}$ are in the volatile (ice) component by number; and that 76 per cent of all the sulphur atoms on $67 \mathrm{P} / \mathrm{C}-\mathrm{G}$ are in the refractory (dust) component by number.

\section{APPENDIX B: A SELECTION OF LINES AND SYNTHETIC SPECTRA OF S-BEARING SPECIES IN THE PILS BAND 7 DATA SET}

All the figures from this appendix are available as supplementary online-only materials.

\section{APPENDIX C: SPECTROSCOPIC LABORATORY INFORMATION}

The data base sources, CDMS or JPL catalogues with their entry numbers, have been provided in the main text for all the species that have been searched for. In addition, the primary references on which these entries are based along with references for the dipole moments for all detected or possibly detected species are given below. Furthermore, the references with laboratory measurements in the range of the PILS survey in cases, in which the primary reference does not cover such data or if these data are an important contribution to the line list are also included.

\section{$\mathrm{C1} \mathrm{SO}_{2}$}

The $v=0$ and $v_{2}=1$ entries for the main isotopic species are based on Müller \& Brünken (2005), and those for ${ }^{34} \mathrm{SO}_{2}$ are based on Belov et al. (1998). The dipole moment of $\mathrm{SO}_{2}$ in several vibrational states was determined by Patel, Margolese \& Dyke (1979).

\section{C2 SO}

The SO entry is based on Bogey et al. (1997). Its dipole moment was measured by Powell \& Lide (1964).

\section{C3 OCS}

The main sources for the OCS entries are Golubiatnikov et al. (2005) for OCS, $v=0$; Morino, Yamada \& Maki (2000) for OCS, $v_{2}=1$; Dubrulle et al. (1980) for $\mathrm{O}^{13} \mathrm{CS}, \mathrm{OC}^{34} \mathrm{~S}$, and $\mathrm{OC}^{33} \mathrm{~S}$. Dipole moment values were determined for OCS in various vibrational states and for several isotopic species by Tanaka, Tanaka \& Suzuki (1985).

\section{C4 CS}

The CS entries are based on Müller et al. (2005), and the main sources of laboratory data are Bogey, Demuynck \& Destombes (1982) and Ahrens \& Winnewisser (1999). The dipole moments of CS in $v=0$ and 1 were measured by Winnewisser \& Cook (1968).

\section{C5 $\mathrm{H}_{2} \mathrm{CS}$}

The $\mathrm{H}_{2} \mathrm{CS}$ data are largely from Maeda et al. (2008), and those of HDCS are from Minowa et al. (1997). Fabricant, Krieger \& Muenter (1977) determined the dipole moment of $\mathrm{H}_{2} \mathrm{CS}$. 


\section{C6 $\mathrm{H}_{2} \mathrm{~S}$}

The $\mathrm{H}_{2} \mathrm{~S}$ entry is based to a considerable extent on Belov et al. (1995). The entries of HDS and $\mathrm{HD}^{34} \mathrm{~S}$ are based on Camy-Peyret et al. (1985). The HDS transition frequencies with microwave accuracy were summarized by Helminger, Cook \& De Lucia (1971). Hillger \& Strandberg (1951) reported a small number of $\mathrm{HD}^{34} \mathrm{~S}$ and HDS transition frequencies. Viswanathan \& Dyke (1984) determined dipole moments of $\mathrm{H}_{2} \mathrm{~S}, \mathrm{HDS}$, and $\mathrm{D}_{2} \mathrm{~S}$.

\section{$\mathrm{C} 7 \mathrm{CH}_{3} \mathrm{SH}$}

The $\mathrm{CH}_{3} \mathrm{SH}$ entry is based on $\mathrm{Xu}$ et al. (2012) with transition frequencies in the range of our survey from Bettens et al. (1999). The information on the dipole moment components was provided by Tsunekawa et al. (1989).

\section{APPENDIX D: ALL THE NON-DETECTED SPECIES}

The one beam offset position of the PILS Band 7 data set was searched for all the sulphur-bearing molecules available in the CDMS catalogue. All those detected are given in Table 1. All those not detected but with a derived upper limit are given in Table 2. The following is a list of all other non-detected species at the $1 \sigma$ level $\left(\sigma=10 \mathrm{mJy}\right.$ beam $^{-1}$ channel $^{-1}$ or $5 \mathrm{mJy}$ beam $^{-1} \mathrm{~km} \mathrm{~s}^{-1}$ ) and their corresponding CDMS entries in brackets: $\mathrm{SO}_{2} v_{2}=1$ (64503), ${ }^{33} \mathrm{SO}_{2}$ (65501), $\mathrm{S}^{18} \mathrm{OO}$ (66502), $\mathrm{S}^{17} \mathrm{OO}(65502), \mathrm{SO} v=1$ (48502), ${ }^{34} \mathrm{SO}(50501),{ }^{33} \mathrm{SO}$ (49501), ${ }^{36} \mathrm{SO}$ (52502), $\mathrm{S}^{18} \mathrm{O}$ (50502), $\mathrm{S}^{17} \mathrm{O}$ (49502), $\mathrm{SO}^{+}$(48010), ${ }^{17} \mathrm{OCS}$ (61504), OC ${ }^{36} \mathrm{~S}$ (64510), ${ }^{18} \mathrm{OC}^{34} \mathrm{~S}(64511),{ }^{18} \mathrm{O}^{13} \mathrm{CS}$ (63503), $\mathrm{O}^{13} \mathrm{C}^{34} \mathrm{~S}$ (63502), $\mathrm{O}^{13} \mathrm{C}^{33} \mathrm{~S}$ (62507), CS $v=0-4$ (44501), $\mathrm{CS}^{+}$ (44512), ${ }^{13} \mathrm{C}^{36} \mathrm{~S}$ (49508), $\mathrm{H}_{2} \mathrm{C}^{34} \mathrm{~S}(48508), \mathrm{H}_{2} \mathrm{C}^{33} \mathrm{~S}$ (47506), $\mathrm{H}_{2}^{13} \mathrm{CS}$ (47505), $\mathrm{H}_{2} \mathrm{~S}$ (34502), $\mathrm{D}_{2} \mathrm{~S}$ (36503), $\mathrm{D}_{2}^{34} \mathrm{~S}$ (38507), cis-HOSO ${ }^{+}$ (65510), $\mathrm{Si}^{34} \mathrm{~S} v=0-2$ (62508), $\mathrm{Si}^{33} \mathrm{~S}$ (61508), $\mathrm{Si}^{36} \mathrm{~S}$ (64514), ${ }^{29} \mathrm{SiS} v=0-2(61506),{ }^{30} \mathrm{SiS} v=0-2(62510),{ }^{29} \mathrm{Si}^{34} \mathrm{~S} v=$ 0,1 (63504), ${ }^{29} \mathrm{Si}^{33} \mathrm{~S}(62512),{ }^{29} \mathrm{Si}^{36} \mathrm{~S}(65507),{ }^{30} \mathrm{Si}^{34} \mathrm{~S} v=0,1$ (64513), ${ }^{30} \mathrm{Si}^{33} \mathrm{~S}(63505),{ }^{30} \mathrm{Si}^{36} \mathrm{~S}(66505), \mathrm{SiS} v=0-5$ (60506), $\mathrm{HSiS}$ (61512), $\mathrm{H}_{2} \mathrm{SiS}$ (62513), OSiS (76517), $\mathrm{HOCS}^{+}$(61510), $\mathrm{HSCO}^{+}$(61509), t-HC(O)SH (62515), c-HC(O)SH (62516), $\mathrm{SH}^{+}$ (33505), HSO (49512), NS $v=1$ (46516), $\mathrm{N}^{34} \mathrm{~S}(48509), \mathrm{N}^{33} \mathrm{~S}$ (47509), $\mathrm{N}^{36} \mathrm{~S}$ (50516), ${ }^{15} \mathrm{NS}$ (47510), DNCS a-type (60510), DNCS b-type (60511), $\mathrm{HN}^{13} \mathrm{CS}$ a-type (60512), $\mathrm{H}^{15} \mathrm{NCS}$ a-type (60513), $\mathrm{HNC}^{34} \mathrm{~S}$ a-type (61519), $\mathrm{C}_{3} \mathrm{~S} v=0$ (68503), $\mathrm{C}_{3} \mathrm{~S} v_{5}=1$ (68505), $\mathrm{C}^{13} \mathrm{CCS}$ (69502), ${ }^{13} \mathrm{CCCS}$ (69503), $\mathrm{CC}^{13} \mathrm{CS}$ (69507), $\mathrm{C}_{3}^{34} \mathrm{~S}(70502), \mathrm{CC}^{13} \mathrm{C}^{34} \mathrm{~S}(71503), \mathrm{C}^{13} \mathrm{CC}^{34} \mathrm{~S}(71504),{ }^{13} \mathrm{CCC}^{34} \mathrm{~S}$ (71505), $\mathrm{C}^{13} \mathrm{C}^{13} \mathrm{CS}$ (70506), ${ }^{13} \mathrm{CC}^{13} \mathrm{CS}$ (70507), $\mathrm{OC}_{3} \mathrm{~S}$ (84502),
$\mathrm{H}_{2} \mathrm{C}_{3} \mathrm{~S}$ (70503), $\mathrm{C}_{4} \mathrm{~S}$ (80501), $\mathrm{C}_{5} \mathrm{~S}$ (92501), ${ }^{13} \mathrm{CC}_{4} \mathrm{~S}$ (93501), $\mathrm{C}^{13} \mathrm{CC}_{3} \mathrm{~S}$ (93502), $\mathrm{C}_{2}^{13} \mathrm{CC}_{2} \mathrm{~S}$ (93503), $\mathrm{C}_{3}^{13} \mathrm{CCS}$ (93504), $\mathrm{C}_{4}^{13} \mathrm{CS}$ (93505), $\mathrm{C}_{5}^{34} \mathrm{~S}$ (94502), CaS $v=0,1$ (72501), ScS (77509), ${ }^{46} \mathrm{TiS}$ (78503), TiS (80505), ${ }^{50} \mathrm{TiS}$ (82503), YS (121502).

The following species have not been detected, because they do not have lines in the frequency range surveyed with PILS Band 7 data: CS $v=1-0,2-1$ (44510), CS $v=2-0(44511), \mathrm{C}^{34} \mathrm{~S}$ $v=1-0$ (46510); ${ }^{13} \mathrm{CS} v=0,1$ (45501); ${ }^{13} \mathrm{CS} v=1-0$ (45509); ${ }^{13} C^{34} \mathrm{~S}(47501),{ }^{13} \mathrm{C}^{33} \mathrm{~S}(47501), \mathrm{H}_{2}^{34} \mathrm{~S}$ (36504), $\mathrm{H}_{2}^{33} \mathrm{~S}$ (35503), $\mathrm{Si}^{34} \mathrm{~S} v=1-0(62509),{ }^{29} \mathrm{SiS} v=1-0(61507),{ }^{30} \mathrm{SiS} v=1-0$ (62511), SiS $v=1-0,2-1$ (60507), SiS $v=2-0$ (60508), $\mathrm{S}$ (32511), $\mathrm{SH}^{-}$(33504), NS $v=1-0$ (46517), ${ }^{15} \mathrm{~N}^{34} \mathrm{~S}$ (49511), HS $v=0$ (33508), HS $v=1$ (33509), $\mathrm{H}^{13} \mathrm{CS}^{+}$(46504), $\mathrm{HSCH}_{2} \mathrm{CN}$ (73503), HCNS (59510), HSNC (59511), $\mathrm{HS}^{13} \mathrm{CN}$ (60514), DSCN (60515), $\mathrm{HSC}^{15} \mathrm{~N}$ (60516), $\mathrm{H}^{34} \mathrm{SCN}$ (61520), $\mathrm{C}_{7} \mathrm{~S}$ (116501).

Finally, the following is a list of all other non-detected species at the $1 \sigma$ level $\left(\sigma=10 \mathrm{mJy}^{\text {beam }}{ }^{-1}\right.$ channel $^{-1}$ or $5 \mathrm{mJy}$ beam ${ }^{-1} \mathrm{~km} \mathrm{~s}^{-1}$ ) that are available only in the JPL catalogue and their corresponding JPL entries in brackets: $\mathrm{MgS}(56009),{ }^{13} \mathrm{CCS}$ (57001), $\mathrm{C}^{13} \mathrm{CS}$ (57002), $\mathrm{CC}^{34} \mathrm{~S}$ (58001), $\mathrm{PS}$ (63007), $\mathrm{SO}_{2} v=2$ (64005), $\mathrm{H}_{2} \mathrm{SO}_{4}$ (98001). SD (34005) does not have lines in the frequency range surveyed with PILS Band 7 data.

\section{APPENDIX E: COMPARISON WITH A MODEL OF HOT CORES}

Efforts on chemical modelling of sulphur networks have recently been revived by Woods et al. (2015). The authors computed the abundances of sulphur-bearing species for hot core conditions upon the inclusion of recent experimental and theoretical data into their chemical network, including a refractory sulphur residue. The modelled molecular ratios (relative to either $\mathrm{H}_{2} \mathrm{~S}$ or OCS; as tabulated in table 9 of Woods et al. (2015) for the standard model with the interstellar cosmic ray ionization rate of $1.3 \times 10^{-17} \mathrm{~s}^{-1}$ ) can be compared to those derived in this work for IRAS 16293-2422 B. The exact values are given in Table E1. An agreement within one order of magnitude is found for OCS and CS relative to $\mathrm{H}_{2} \mathrm{~S}$, and for $\mathrm{H}_{2} \mathrm{~S}$ relative to OCS. The modelled ratios for $\mathrm{H}_{2} \mathrm{CS}$ are three orders of magnitude higher, which may be explained by the fact that the grain-surface network used in the models is fairly small and the grain-surface chemistry is not accounted for in full. $\mathrm{H}_{2} \mathrm{CS}$ is expected to be involved in many grain-surface reactions and will likely be used up for synthesis of larger sulphur-bearing species, such as $\mathrm{CH}_{3} \mathrm{SH}$ (analogous to the sequential hydrogenation of $\mathrm{CO}$ leading to $\mathrm{CH}_{3} \mathrm{OH}$ with $\mathrm{H}_{2} \mathrm{CO}$ as an intermediate).

Table E1. Molecular ratios relative to $\mathrm{H}_{2} \mathrm{~S}$ and OCS as measured with these interferometric ALMA observations at the one beam offset position from source B of IRAS $16293-2422^{a}$ in comparison to hot core models ${ }^{b}$.

\begin{tabular}{|c|c|c|c|c|}
\hline \multirow[t]{2}{*}{ Species } & \multicolumn{2}{|c|}{ Molecular ratios relative to $\mathrm{H}_{2} \mathrm{~S}$ (per cent) } & \multicolumn{2}{|c|}{ Molecular ratios relative to OCS (per cent } \\
\hline & ALMA B & Model & ALMA B & Model \\
\hline $\mathrm{H}_{2} \mathrm{~S}$ & 100 & 100 & $68-679$ & 29 \\
\hline OCS & $147-15$ & 341 & 100 & 100 \\
\hline SO & $0.3-0.03$ & 58 & 0.2 & 17 \\
\hline $\mathrm{SO}_{2}$ & $0.8-0.08$ & $1.5 \times 10^{4}$ & 0.5 & $4.5 \times 10^{3}$ \\
\hline $\mathrm{CS}$ & $2-0.2$ & 1.2 & 2 & 0.3 \\
\hline $\mathrm{H}_{2} \mathrm{CS}$ & 0.8 & 129 & 0.5 & 38 \\
\hline
\end{tabular}

Notes. ${ }^{a}$ Values taken from Table 3.

${ }^{b}$ Table 9 of Woods et al. (2015) for the standard model with the interstellar cosmic ray ionization rate of $1.3 \times 10^{-17} \mathrm{~s}^{-1}$. 
For $\mathrm{SO}$ and $\mathrm{SO}_{2}$, the differences are many orders of magnitude between the observed and modelled values of Woods et al. (2015). This likely has to do with the fact that the models have been run for a very long time of $10^{7} \mathrm{yr}$, which implies that many gaseous species are driven into $\mathrm{SO}$ and $\mathrm{SO}_{2}$, leading to their overproduction. At earlier times (as seen in fig. 6 of Woods et al. 2015), the model results have a closer agreement for $\mathrm{SO}$ with observations. $\mathrm{SO}_{2}$ remains too high nevertheless, which may have to do with thermal desorption being calculated via an efficiency factor. It seems that the modelling approach of Woods et al. (2015) is insufficient at reproducing the sulphur chemistry towards IRAS 16293-2422 B. The differences with cometary ROSINA measurements are just as large. The development of a full gas-grain chemical network for sulphur, including grain-surface chemistry, is the topic of future research. For the case of dark prestellar core, Vidal et al. (2017) have been able to successfully reproduce observed abundances of sulphur-bearing species without the need for artificial sulphur depletion.

A comparison with observations of hot cores such as Sgr B2(N), Sgr B2(M) (e.g. Belloche et al. 2013), and Orion KL (e.g. Esplugues et al. 2014) is beyond the scope of this paper due to the mandatory discussion on emitting regions and structures sampled with observations. Future high spatial resolution, ALMA observations will mediate this problem.

This paper has been typeset from a $\mathrm{T}_{\mathrm{E}} \mathrm{X} / \mathrm{LT}_{\mathrm{E}} \mathrm{X}$ file prepared by the author. 